\title{
Vehicle-Component Identification Based on Multiscale Textural Couriers
}

\author{
William Wai Leung Lam, Clement Chun Cheong Pang, Member, IEEE, and \\ Nelson Hon Ching Yung, Senior Member, IEEE
}

\begin{abstract}
This paper presents a novel method for identifying vehicle components in a monocular traffic image sequence. In the proposed method, the vehicles are first divided into multiscale regions based on the center of gravity of the foreground vehicle mask and the calibrated-camera parameters. With these multiscale regions, textural couriers are generated based on the localized variances of the foreground vehicle image. A new scale-space model is subsequently created based on the textural couriers to provide a topological structure of the vehicle. In this model, key feature points of the vehicle can significantly be described based on the topological structure to determine the regions that are homogenous in texture from which vehicle components can be identified by segmenting the key feature points. Since no motion information is required in order to segment the vehicles prior to recognition, the proposed system can be used in situations where extensive observation time is not available or motion information is unreliable. This novel method can be used in real-world systems such as vehicle-shape reconstruction, vehicle classification, and vehicle recognition. This method was demonstrated and tested on 200 different vehicle samples captured in routine outdoor traffic images and achieved an average error rate of $6.8 \%$ with a variety of vehicles and traffic scenes.
\end{abstract}

Index Terms-Feature-point extraction, image segmentation, texture analysis, vehicle-component identification, vehicle occlusion.

\section{INTRODUCTION}

$\mathbf{T}$ HE INVESTIGATION of -D objects from single monocular intensity images is one of the fundamental problems in image analysis and computer vision. Recently, research studies of vehicle-shape reconstruction [4], vehicle classification [1], [6], [9], [19], and vehicle recognition [16], [18] have become the most important areas in visual traffic surveillance. Most of these studies, however, fail to distinguish vehicles with similar dimensions due to the lack of other details. In visual traffic surveillance, most vehicle-recognition systems simply distinguish the size, dimension, shape, or contour of the vehicle, from which vehicles can be categorized by type. Broadly, these techniques addressed the problem of classifying vehicles of the same size or dimension. If different vehicles of similar dimen-

Manuscript received May 4, 2006; revised February 28, 2007; accepted May 20, 2007. This work was supported in part by the Research Grants Council of the Hong Kong Special Administrative Region under Grant HKU7198/05E and in part by the Postgraduate Studentship of The University of Hong Kong. The Associate Editor for this paper was C. Stiller.

The authors are with the Laboratory for Intelligent Transportation Systems Research, Department of Electrical and Electronic Engineering, The University of Hong Kong, Hong Kong (e-mail: wllam@eee.hku.hk; ccpang@eee.hku.hk; nyung@eee.hku.hk).

Color versions of one or more of the figures in this paper are available online at http://ieeexplore.ieee.org.

Digital Object Identifier 10.1109/TITS.2007.908144

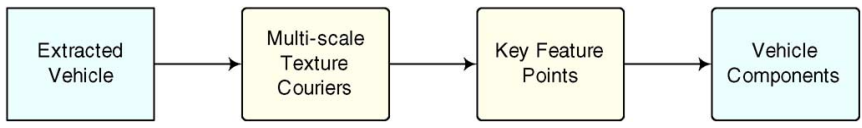

Fig. 1. Identification procedures of vehicle components.

sions are present, these methods become inadequate, as similarsized vehicles will most likely be recognized as one class because of the nonutilization of vehicle details, and consequently, the reconstructed model of vehicle shape will also be identical.

To accomplish these difficult tasks in a real-world environment, it appears necessary to use some advanced imageprocessing methods to explore the intrinsic information of the vehicle. Recently, high-tech surveillance cameras have been emerging and providing high-resolution images. This motivates us to consider decomposing the vehicle object into components such as roof, bonnet, windscreen, etc. as an alternative way to discriminate between different vehicles.

To resolve many of these problems, we propose a method, as shown in Fig. 1. Assuming that the vehicle is in motion, we first employ the extraction method, as described in [11]. From each extracted vehicle, we then estimate its dimension [9] to identify similar-sized vehicles and to compute its textural couriers based on the centers of gravity of multiscale regions and the localized variance values of the foreground vehicle image. By fusing textural regions corresponding to a selected scale, key feature points are estimated based on the topological structure of the textural couriers. Subsequently, vehicle components can be identified by segmenting those key feature points.

A brief problem analysis is given in the next section, which is followed by an overview of the recent related research studies in Section III. Details of the proposed method are presented in Section IV, simulation results and discussions are specified in Section V, suggestions of further development are given in Section VI, and conclusions are drawn in Section VII.

\section{Problem Analysis}

Typically, research development of visual traffic surveillance can be considered in two major areas: the well-developed area and the developing area. As shown in Fig. 2, the well-developed area includes the techniques of background estimation, camera calibration, vehicle segmentation, deformable modeling, and occlusion detection, as indicated as solid-line diagrams. These techniques can simply utilize image-processing algorithms and geometry parameters to achieve fulfilled results. Beyond this area, researchers are currently investigating the developing area, which includes the techniques of vehicle-shape 


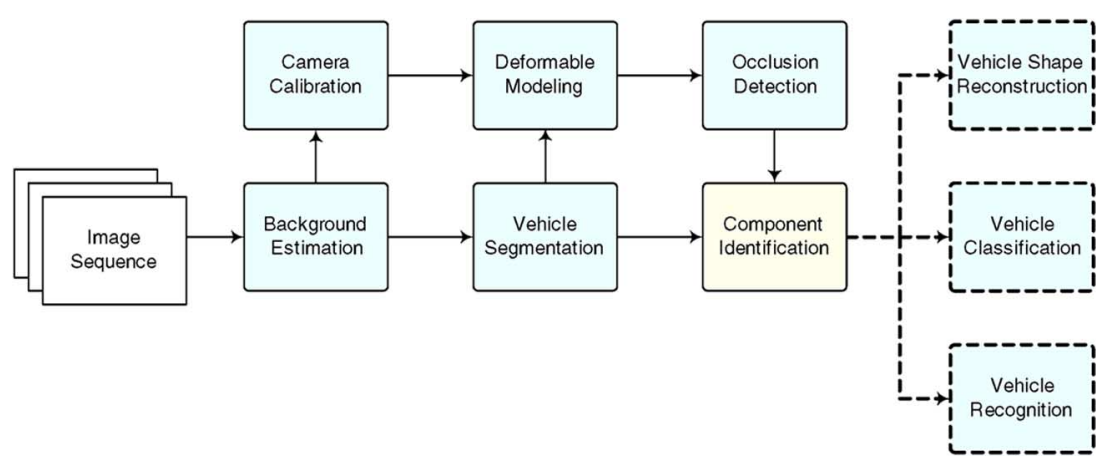

Fig. 2. Conceptual diagram of visual traffic surveillance systems.

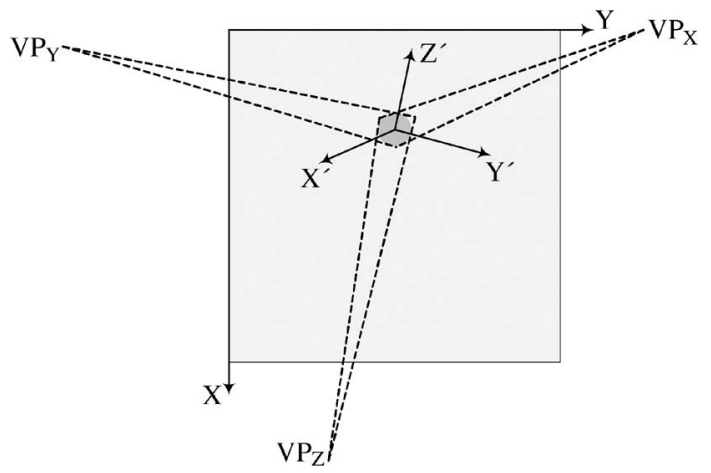

Fig. 3. Three-dimensional cuboid model.

reconstruction, vehicle classification, vehicle recognition, and other advanced post-analysis processes of an image sequence, as indicated by dashed-line diagrams. These techniques require timely track data of image sequence and detailed contents of the vehicle in order to attain more precise results. Our technique of component identification would be the major role between these well-developed and developing areas as we can contribute further distinctive characteristics. For that reason, the utilization of vehicle details would lead to much better achievement of those techniques in the developing area.

As the technique of component identification is a critical issue in visual traffic surveillance, we should further investigate the challenges of this technique. In many research studies, vehicles could be categorized based on the length of vehicle. To calculate the vehicle length, a simple 3-D cuboid model can be employed to fit the vehicle, as shown in Fig. 3 .

A foreground vehicle mask is symbolized as a shaded circle, and the dimension can be calculated by finding the edges and vertices with a calibrated-camera model [9]. An example developed in [9] is shown in Fig. 4, as the shape of the bus is in the vicinity of the cuboid; thus, we can define point $V_{7}$ as the intersection point which is extended from vanishing point $\mathrm{VP}_{X}$ to vertex $V_{6}, \mathrm{VP}_{Y}$ to vertex $V_{2}$, and $\mathrm{VP}_{Z}$ to vertex $V_{4}$, as shown in Fig. 4(a). Along with point $V_{7}$, an individual component can easily be defined within one of those quadrangles. For instance, the edge between the roof and side windows of the bus can be aligned on line $V_{6} V_{7}$. However, many small vehicles are not in the shape of cuboid. Fig. 4(b) shows an example of a sedan; if we also define $V_{7}$ as the intersection, there is no help in identifying the individual component, such as the edge between the roof and side windows, as it might not be aligned on line $V_{6} V_{7}$. Therefore, our challenges and focus of this paper

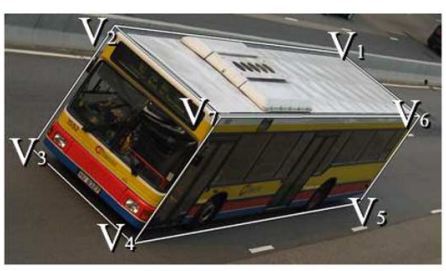

(a)

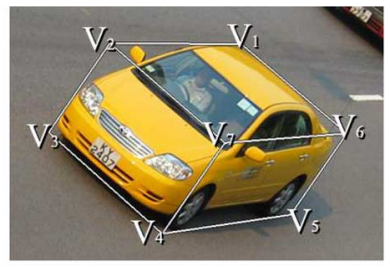

(b)
Fig. 4. Deformable models of bus and sedan. (a) Large vehicle. (b) Small vehicle.

would mainly be on identifying the vehicle components of small and streamlined vehicles.

\section{RELATED WORKS}

Generally, in visual traffic surveillance, many research studies addressed unsatisfied results due to the lack of classifying component details of same-sized vehicles, as shown in Fig. 2. In vehicle-classification [1], [6], [9], [19] and vehicle-recognition systems [16], [18], vehicles could only be identified into either large or small vehicles or into predefined vehicle models, and no further classified or recognized components could be made.

Avery et al. [1] presented an image-processing algorithm for length-based vehicle classification, with an image stream captured by an uncalibrated video camera. The basis of the algorithm was to relatively compare vehicle lengths with each other to estimate long vehicles. Gupte et al. [6] modeled vehicles as rectangular patches to estimate vehicle parameters with a Kalman filter, and afterward, a nonrigid model-based approach was used to classify vehicles. Lai et al. [9] extracted moving vehicles from traffic image sequences and fitted them with a simple deformable vehicle model. By using a set of coordination mapping functions derived from a calibrated-camera model and relying on a shadow removal method, vehicle dimensions were estimated. Wei et al. [19] classified vehicles by using a parameterized model, as well as neural networks, which describe the features of the vehicle. In this model, vertices and their topological structure were regarded as the key features, and vehicles were recognized based on multilayer perception networks.

Sidla et al. [16] presented an appearance-based pattern matching algorithm which relied on principal component analysis (PCA) to detect and classify cars and trucks under geometric constraints. Tan and Baker [18] extracted vehicle by matching model discrimination. The algorithms eliminated the need of 
explicit symbolic feature extraction and matching. The pose and class of an object were determined by a form of voting and 1-D correlations directly based on image gradient data.

In vehicle-shape-reconstruction system, Fung et al. [4] additionally considered vehicle shape for classification based on vehicle motion. A 3-D vehicle-shape information from a 2-D monocular image sequence was recovered by extracting stable feature points, corresponding feature points between frames, computing the heights from projected displacements, and using the forward and backward image-to-world coordinate mapping function. However, the dimension estimation error might be caused by the accuracy of the feature-correspondence method.

\section{Proposed Method}

In the proposed method, it is assumed that the surveillance camera is road-side-mounted and stationary, whereas the light source can be single and strong, as in daytime, or can be multiple and diffused, as in nighttime. Based on these assumptions, the proposed method comprises the following: 1) multiscale textural-courier construction and 2) key-feature-point estimation.

\section{A. Overview}

In general, a visual traffic surveillance system can be shown in Fig. 2. In the figure, background estimation estimates the background image from the image by averaging out the moving objects from the image. A "scoreboard algorithm" from [10] is employed in this paper for the background estimation as it is fast and accurate for the estimation of the stationary background. The basic idea of this algorithm is that it keeps track of the pixel variations in the image sequence to select between the running-mode and the running-average algorithms in each estimation in order to optimize both the speed and accuracy of the algorithm. With the estimated background image, it is possible to determine a set of camera parameters such as pan angle, tilt angle, swing angle, camera distance, and focal length from the background image through the process of camera calibration. The camera-calibration method proposed by Fung et al. [5] employed road-lane markings which were readily found in typical traffic scene as the calibration pattern. The proposed method utilized the geometric properties of the endpoints of the roadlane markings to determine the required camera parameters that governed the mapping relationship between the 2-D image and 3-D world coordinates. Vehicle segmentation segments the vehicles from the input image based on the background image. The texture-based vehicle-segmentation approach described in [11] is employed to extract the moving vehicles due to its ability to accurately generate a vehicle mask with the shadow, as well as the reflection on the vehicle chassis excluded from the segmentation. The basic idea of this method is to consider the differences in texture, luminance, and chrominance between the input and background frames in order to form three likelihood maps and to perform a logical OR operation on the likelihood maps followed by the morphological operations to produce a foreground mask that represents the segmented vehicle. Deformable modeling fits a 2 -D projection of a simple
3-D cuboid model onto a binary representation of the segmented vehicle based on the geometric property of the extracted vehicle mask. The projection is done by a set of coordination mapping functions derived from a calibrated-camera model [9] based on the camera parameters. This set of calibrated-camera parameters is useful in providing the transformation between the 3-D world and 2-D image coordinates, as seen by the camera. After model fitting, the cuboid model is transformed from the 2-D image coordinates back into the 3-D world coordinates in order to determine the width, length, and height of the vehicle [9]. This estimation method enables us to identify the vehicles of similar dimensions for identification in the next step. Occlusion detection determines whether there is an occlusion inside the fitted 3-D cuboid model. The method that calculates the area ratio between the binary vehicle mask and the cuboid model is used to detect occlusion due to its simplicity. Occlusion is detected when the area ratio exceeds a predefined value, which indicates that the model is fitted onto more than one vehicle since there is a large gap between the vehicle mask and the fitted model due to occlusion. In this paper, our focus is on proposing a new method for component identification, which provides more significant vehicle features for further advanced research studies in visual traffic surveillance.

\section{B. Vehicle-Component Identification}

Apart from vehicle-component identification, the most common ways of finding the features of vehicles are based on brightness, color, corners, and edges of the vehicle body. For the brightness and color criteria, different styles of vehicle may contain different values of brightness and color. For instance, if the vehicle is black in color, that will not be an easy task to separate vehicle body and wheels. For the corners and edges criteria, they require knowledge-based methods to distinguish their features. For instance, if we find the edges of streamlined sedan [Fig. 18(a)] by Canny edge detection (as shown in Fig. 5), the simulated result shows that there are too many undesired edges. If we have no human knowledge of the vehicle model and camera-calibration parameters, it is extremely harsh to define which edges correspond to which component boundaries.

Therefore, our challenges would mainly be on identifying vehicle components by segmenting the component boundaries. Based on our observation on traffic image sequences, different kinds of vehicle may contain different components. For example, a roof window or an antenna can be optional for vehicles. In such situation, we have to define certain vehicle features that are robust enough to segment components, and therefore, we based on typical traffic scenes, as shown in Fig. 6, to define the three key feature points $K_{1}, K_{2}$, and $K_{3}$ of the vehicle that is coming toward the camera, as shown in Table I.

Our decisions of making these three key feature points are mainly based on the characteristics of vehicles that most vehicles have windscreen and headlights, except for motorcycles. For the motorcycle case, it can initially be classified by computing vehicle dimensions, as mentioned in [9]. The robustness of these key feature points can be adapted from the different kinds of vehicle such as sedan, jeep, hatchback, van, minibus, and bus. All of them contain these key feature points, and 


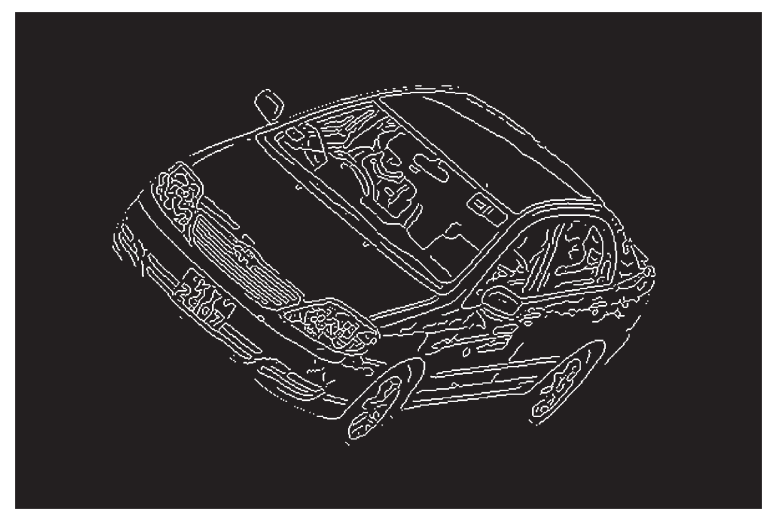

Fig. 5. Canny edge detection.

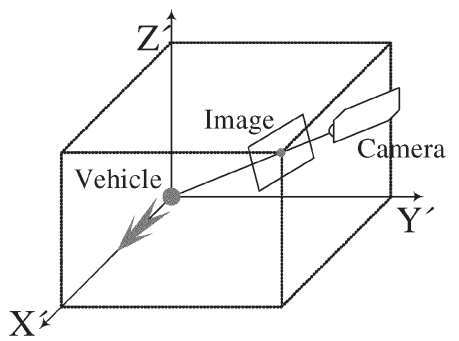

Fig. 6. Camera model of visual traffic surveillance.

TABLE I

DeFinition of Key FeAture Points

\begin{tabular}{|c|c|}
\hline Key Feature Point & Definition \\
\hline $\mathrm{K}_{1}$ & $\begin{array}{c}\text { Intersects with roof, windscreen } \\
\text { and side windows }\end{array}$ \\
\hline $\mathrm{K}_{2}$ & $\begin{array}{c}\text { Intersects with windscreen, side windows, bonnet } \\
\text { and door panels }\end{array}$ \\
\hline $\mathrm{K}_{3}$ & $\begin{array}{c}\text { Intersects with bonnet, } \\
\text { grille and fender }\end{array}$ \\
\hline
\end{tabular}

vehicle components can further be decomposed by segmenting through these key feature points. The estimation of key feature points requires the technique of constructing multiscale textural couriers, which will be discussed in the next section.

After we search out the key feature points $K_{1}, K_{2}$, and $K_{3}$, the next step is to identify the major component-region boundaries. In the proposed method, the component-region boundaries are computed by subdividing six diverse component regions through the vanishing points $\mathrm{VP}_{X}, \mathrm{VP}_{Y}$, and $\mathrm{VP}_{Z}$, as shown in Fig. 7. Fig. 7(a) shows an image with a foreground vehicle contour, whereas Fig. 7(b) shows the dashed lines which are indicated as straight lines elongated through the vanishing points $\mathrm{VP}_{X}, \mathrm{VP}_{Y}$, and $\mathrm{VP}_{Z}$, as developed in [9].

Fig. 7(c) shows the defined key feature points $K_{1}, K_{2}$, and $K_{3}$ of vehicle, whereas the component-region boundaries can be computed by extending the key feature points $K_{1}, K_{2}$, and $K_{3}$ to the vanishing points $\mathrm{VP}_{X}, \mathrm{VP}_{Y}$, and $\mathrm{VP}_{Z}$, as shown in Fig. 7(d). With the component-region boundaries, the components of regions $R_{\mathrm{R}}, R_{\mathrm{W}}, R_{\mathrm{B}}, R_{\mathrm{G}}, R_{\mathrm{S}}$, and $R_{\mathrm{D}}$ can be defined in Table II.

\section{Multiscale Textural-Courier Construction}

As shown in Fig. 2, the vehicle-segmentation process segments the vehicles from the input image based on the background image. The texture-based vehicle-segmentation approach described in [11] is employed to extract the moving vehicles. If occlusion detection determines that there is no occlusion inside the fitted 3-D cuboid model, the componentidentification process can take place by starting with the construction of the multiscale textural couriers.

After the vehicles have been segmented, the vehicle mask $O_{\mathrm{V}}$ of each similar-sized vehicle is constructed by segmenting the boundary of the vehicle through the subtraction between the input frame $f_{\mathrm{i}}$ and the stationary background frame $f_{\mathrm{b}} . O_{\mathrm{V}}$ is defined as the root region and is symbolized as gray ellipse in Fig. 8.

The scale level $L$ of the root region is classified as scale 0 , and therefore, the vehicle mask $O_{\mathrm{V}}$ can be written as $R^{0}$, which is also defined as scale 0 of division $X\left(R_{X}^{0}\right)$ and division $Y\left(R_{Y}^{0}\right)$. The next step is to calculate the next-scale bisection regions of division $X$ with locations $m=0$ and $m=1$ as $R_{X_{0}}^{1}$ and $R_{X_{1}}^{1}$, respectively, as shown in Fig. 8(a). The bisection regions of division $Y$ with locations $n=0$ and $n=1$ as $R_{Y_{0}}^{1}$ and $R_{Y_{1}}^{1}$, respectively, are shown in Fig. 8(b).

As shown in Fig. 8(a), the subregions $R_{X_{0}}^{1}$ and $R_{X_{1}}^{1}$ are separated by the vector $\overrightarrow{\mathrm{VP}_{Y} C_{X}^{0}}$ as the boundary, which is constructed by connecting the vanishing point $\mathrm{VP}_{Y}$ and the central pixel $C_{X}^{0}$ of region $R_{X}^{0}$, where the central pixel $C_{X_{m}}^{L}$ of every individual region $R_{X_{m}}^{L}$ can be calculated by the center of gravity as

$$
C_{X_{m}}^{L}=\frac{\sum_{i, j \in R_{X_{m}}^{L}} i \cdot O_{\mathrm{V}}(i, j)}{\sum_{i, j \in R_{X_{m}}^{L}} O_{\mathrm{V}}(i, j)}
$$

where $m$ is the region location from 0 to $2^{L}-1, i$ and $j$ are the position displacements within the vehicle mask $O_{\mathrm{V}}$, and $L$ is the region scale level. Mathematically, the bisection regions $R_{X_{2 m}}^{L+1}$ and $R_{X_{2 m+1}}^{L+1}$ of next scale level $L+1$ can vertically be divided along with $\mathrm{Y}^{\prime}$ as

$$
\begin{gathered}
R_{X_{2 m}}^{L+1}(i, j)=R_{X_{m}}^{L}\left(i^{\prime}, j^{\prime}\right), \quad \text { if } \quad i^{\prime} \leq C_{X_{m}}^{L} \\
R_{X_{2 m+1}}^{L+1}(i, j)=R_{X_{m}}^{L}\left(i^{\prime}, j^{\prime}\right), \quad \text { if } \quad i^{\prime}>C_{X_{m}}^{L}
\end{gathered}
$$

where $i^{\prime}$ and $j^{\prime}$ are the position displacements of the 3-D coordinates projected on the image. Fig. 9(a) shows the concept of locating the subregions $R_{X_{2 m}}^{L+1}$ and $R_{X_{2 m+1}}^{L+1}$ and the central pixel $C_{X_{m}}^{L}$ of each individual region $R_{X_{m}}^{L}$.

With the same approach of division $X$, the subregions $R_{Y_{0}}^{1}$ and $R_{Y_{1}}^{1}$ are separated by the vector $\overrightarrow{\mathrm{VP}_{X} C_{Y}^{0}}$ as the boundary, which is constructed by connecting the vanishing point $\mathrm{VP}_{\mathrm{X}}$ and the center pixel $C_{Y}^{0}$ of region $R_{Y}^{0}$, as shown in Fig. 8(b). The central pixel $C_{Y_{n}}^{L}$ of every individual region $R_{Y_{n}}^{L}$ can be calculated by the center of gravity as

$$
C_{Y_{n}}^{L}=\frac{\sum_{i, j \in R_{Y_{n}}^{L}} i \cdot O_{\mathrm{V}}(i, j)}{\sum_{i, j \in R_{Y_{n}}^{L}} O_{\mathrm{V}}(i, j)}
$$

where $n$ is the region location from 0 to $2^{L}-1, i$ and $j$ are the position displacements within the vehicle mask $O_{\mathrm{V}}$, and $L$ 


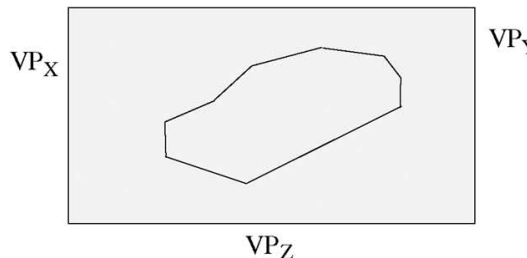

(a)

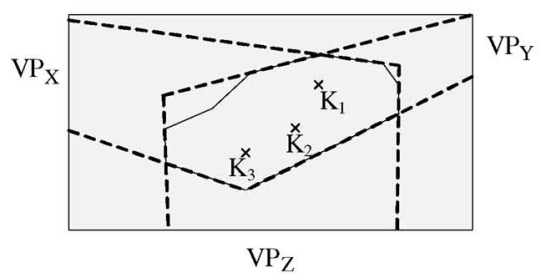

(c)

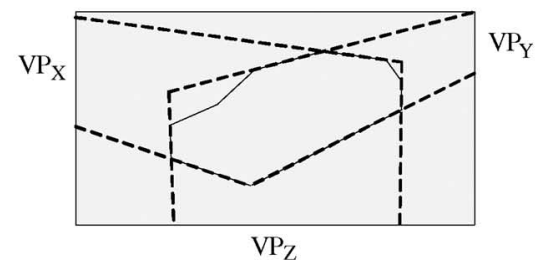

(b)

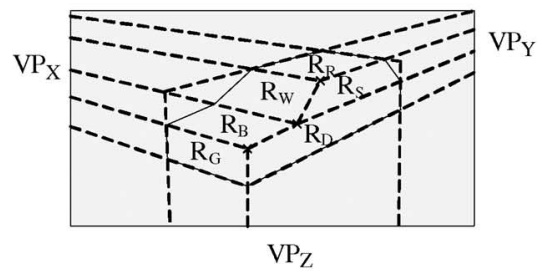

(d)

Fig. 7. Vehicle boundaries of common vehicle. (a) Vehicle contour. (b) 3-D cuboid. (c) Key feature points. (d) Segmented component regions.

TABLE II

DEFINED VEHICLE COMPONENTS

\begin{tabular}{|c|c|}
\hline Region & Components \\
\hline $\mathrm{R}_{\mathrm{R}}$ & Roof \\
\hline $\mathrm{R}_{\mathrm{W}}$ & Windscreen \\
\hline $\mathrm{R}_{\mathrm{B}}$ & Bonnet \\
\hline $\mathrm{R}_{\mathrm{G}}$ & Grille, Headlight, Bumper \\
\hline $\mathrm{R}_{\mathrm{S}}$ & Side Windows \\
\hline $\mathrm{R}_{\mathrm{D}}$ & Door Panels, Fender, Wheel \\
\hline
\end{tabular}

is the region scale level. Mathematically, the bisection regions $R_{Y_{2 n}}^{L+1}$ and $R_{Y_{2 n+1}}^{L+1}$ of the next scale level $L+1$ can horizontally be divided along with $X^{\prime}$ as

$$
\begin{array}{cc}
R_{Y_{2 n}}^{L+1}(i, j)=R_{Y_{n}}^{L}\left(i^{\prime}, j^{\prime}\right), & \text { if } \quad i^{\prime} \leq C_{Y_{n}}^{L} \\
R_{Y_{2 n+1}}^{L+1}(i, j)=R_{Y_{n}}^{L}\left(i^{\prime}, j^{\prime}\right), & \text { if } \quad i^{\prime}>C_{Y_{n}}^{L}
\end{array}
$$

where $i^{\prime}$ and $j^{\prime}$ are the position displacements of the 3-D coordinates projected on the image. Fig. 9(b) shows the concept of locating the subregions $R_{Y_{2 n}}^{L+1}$ and $R_{Y_{2 n+1}}^{L+1}$ and the central pixel $C_{Y_{n}}^{L}$ of each individual region $R_{Y_{n}}^{L}$.

Finally, the multiscale regions $R_{m, n}^{L}$ can be formulated by intersecting the division $X$ regions $R_{X_{m}}^{L}$ and the division $Y$ regions $R_{Y_{n}}^{L}$ as

$$
R_{m, n}^{L}=R_{X_{m}}^{L} \cap R_{Y_{n}}^{L} .
$$

If $O_{\mathrm{V}}$ is defined as the root region $R^{0}$, as shown in Fig. 8, then $R^{0}$ can be divided into four scale 1 subregions $R_{0,0}^{1}, R_{0,1}^{1}$, $R_{1,0}^{1}$, and $R_{1,1}^{1}$, as shown in Fig. 10. This illustrates the concept of partitioning $O_{\mathrm{V}}$ into the multiscale regions $R_{m, n}^{L}$.

The purpose of construing the multiscale regions is to equalize the area of each region within the same scale level while keeping the localization features. After partitioning $O_{\mathrm{V}}$ into the multiscale regions $R^{L}$, the intensity courier $R_{I_{m, n}}^{L}$ of each subregion $R_{m, n}^{L}$ is determined based on intensity averaging

$$
R_{I_{m, n}}^{L}=\frac{\sum_{i, j \in R_{m, n}^{L}} f_{\mathrm{i}}(i, j)}{\sum_{i, j \in R_{m, n}^{L}} O_{\mathrm{V}}(i, j)}
$$

and the textual courier $R_{T_{m, n}}^{L}$ of each subregion $R_{m, n}^{L}$ is calculated based on intensity variance

$$
R_{T_{m, n}}^{L}=\frac{\sum_{i, j \in R_{m, n}^{L}}\left(f_{\mathrm{i}}(i, j)-R_{I_{m, n}}^{L}\right)^{2}}{\sum_{i, j \in R_{m, n}^{L}} O_{\mathrm{V}}(i, j)} .
$$

If the textual courier $R_{T_{m, n}}^{L}$ does not occupy any pixel $f_{\mathrm{i}}(i, j)$ within the vehicle mask $O_{\mathrm{V}}$, this textual courier $R_{T_{m, n}}^{L}$ is called null courier which has no any value. At scale zero, the textual courier $R_{T}^{0}$ is the largest and continues to decrease in subsequent scales until $R_{T_{m, n}}^{L}$ becomes zero for the smallest subregion $R_{m, n}^{L}$, as shown in Fig. 11. These squarelike multiscale regions are useful in comparing visual properties between vehicle components of inconsistent sizes. Compared with the traditional edge detector of segmentation, our textural couriers $R_{T}^{L}$ are helpful in sorting out vehicle components as each vehicle component has a comparative textural relationship with others. This is significantly helpful in judging edges that are capable of being elongated to the vanishing points $\mathrm{VP}_{X}$ and $\mathrm{VP}_{Y}$, so that our defined key feature points can be estimated based on these relationships.

The process of estimating the key feature points will take place at one of the textual-courier $R_{T}^{L}$ scale levels. This scale level is called as the critical scale $C$, which has comparative textural properties for the analysis of the key feature points. To justify the critical scale $C$, we have to calculate the number $T^{L}$ of the textual couriers $R_{T_{m, n}}^{L}$ that have zero values and the total number $(m+1)(n+1)$ of subregions $R_{m, n}^{L}$ within the same scale level. Subsequently, we find their ratio $S^{L}$ as

$$
S^{L}=\frac{T^{L}}{(m+1)(n+1)}
$$

where $m$ and $n$ are the region locations from 0 to $2^{L}-1$. To find a suitable scale for the estimation of the key feature points, we define the critical textural scale $C$, which is chosen when the difference between $S^{L+1}$ and $S^{L}$ is maximum

$$
C=L, \quad \text { if } \max \left(S^{L+1}-S^{L}\right) .
$$




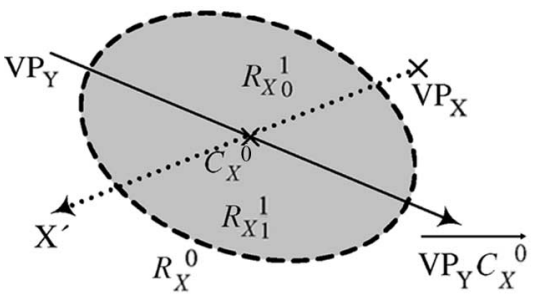

(a)

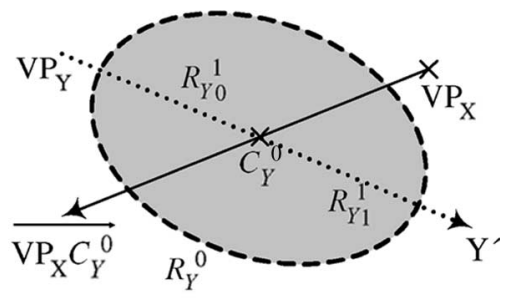

(b)

Fig. 8. Root region $R^{0}$. (a) $R_{X}^{0}$. (b) $R_{Y}^{0}$.

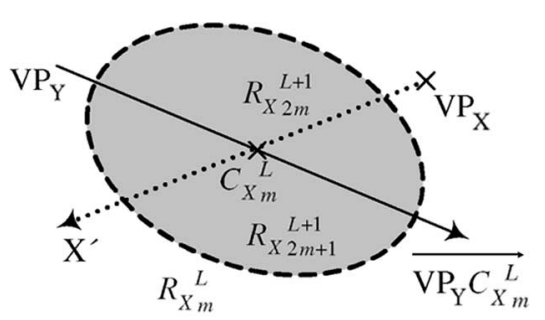

(a)

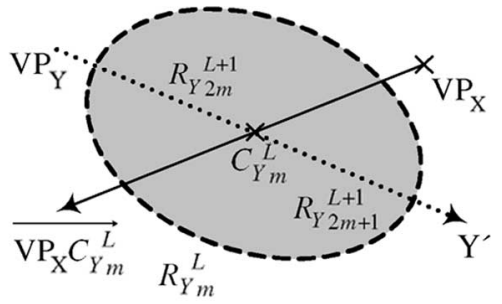

(b)

Fig. 9. Bisection regions. (a) $R_{X_{m}}^{L}$. (b) $R_{Y_{m}}^{L}$.

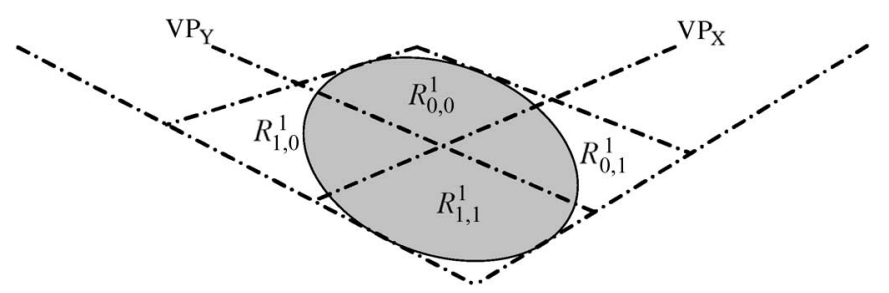

Fig. 10. Scale 1 regions.
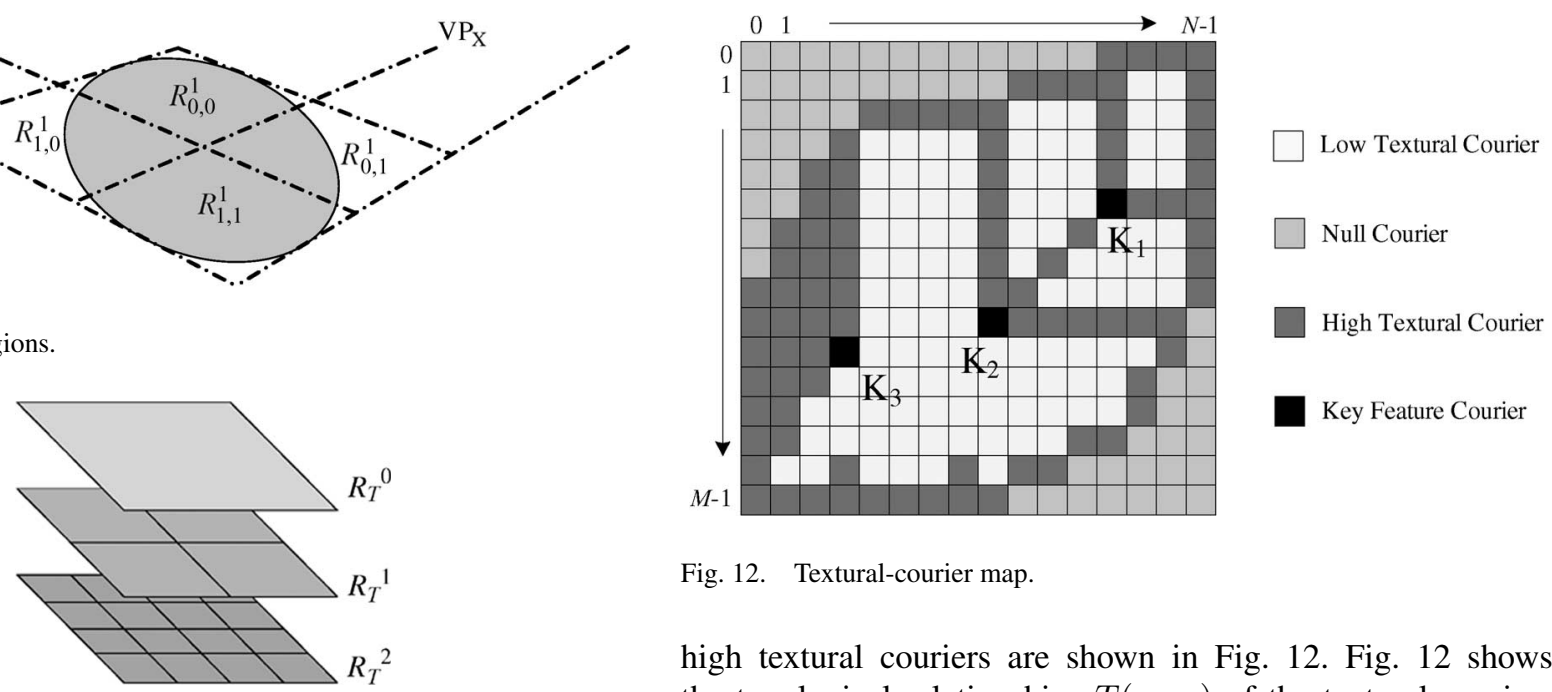

Fig. 12. Textural-courier map.

high textural couriers are shown in Fig. 12. Fig. 12 shows the topological relationships $T(m, n)$ of the textural couriers of typical vehicles with scale level 4 , where $M=2^{L}$, and $N=2^{L}$. For simplicity, the low textural courier is demonstrated as a white-color square, whereas the high textural courier is demonstrated as a dark-gray-color square, the null courier is demonstrated as a light-gray-color square, and the three key feature points are demonstrated as black-color squares and defended as key feature couriers.

As shown in Table I, $K_{1}$ intersects with the roof, windscreen, and side windows, and therefore, there is an $L$ sharp of high textural couriers, indicating a vertical boundary between the roof and windscreen and a horizontal boundary between the roof and side windows. $K_{2}$ has similar properties as $K_{1}$; it intersects with the windscreen, side windows, bonnet, and door panels so that there is also an $L$ sharp of high textural couriers, indicating a vertical boundary between the windscreen and grille and a horizontal boundary between the side windows and door panels. For $K_{3}$, it intersects with the grille, bonnet, and fender, and therefore, it lies between the region of high textural couriers with grille and the region of low textural 


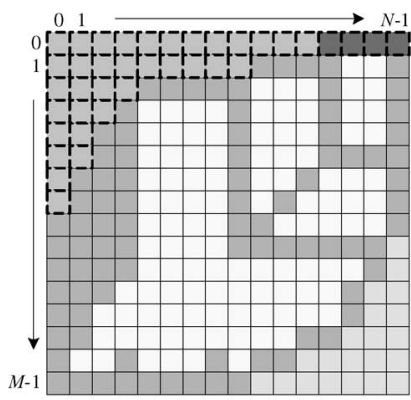

(a)

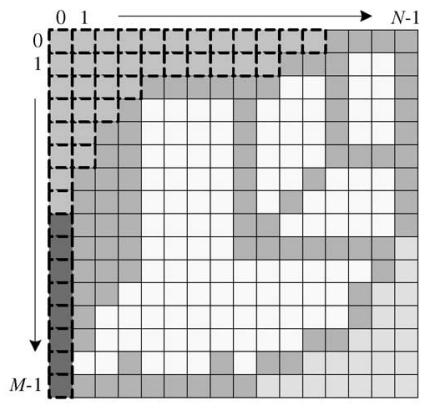

(b)
Fig. 13. Textural-courier map. (a) Length of roof. (b) Width of vehicle.

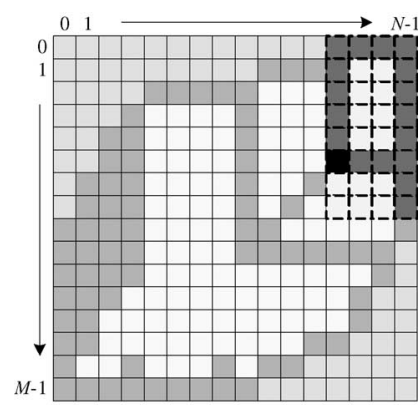

(a)

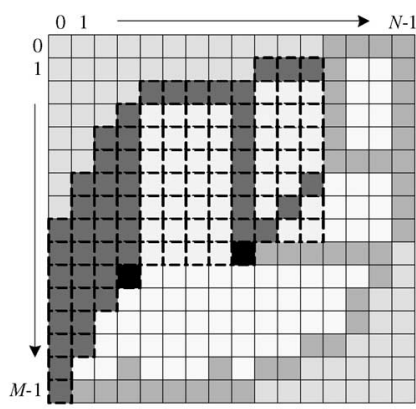

(b)
Fig. 14. Textural couriers map. (a) Location of $K_{1}$. (b) Locations of $K_{2}$ and $K_{3}$.

couriers with bonnet and fender. Based on these observations, the key feature couriers can be evaluated in the following procedures.

1) Evaluate the length of the roof and the width of the vehicle.

2) Search the key feature courier $K_{1}$.

3) Search the key feature couriers $K_{2}$ and $K_{3}$.

To demonstrate the procedures of finding the key feature points, we assume the high textural courier $T(m, n)$ as value 1 and the low textural courier $T(m, n)$ as value 0 . We first find the key feature couriers by finding the length of the roof $l_{\mathrm{r}}$ and the width of the vehicle $w_{\mathrm{V}}$. As we can see, the length of the roof $l_{\mathrm{r}}$ approximately equals the length of the regions that contain the most nonnull couriers at the first row $(m=0)$, as shown in Fig. 13(a), with dark gray squares, and we define the length of roof $l_{\mathrm{r}}$ starting from $n=12$ to $n=N-1$ in this demonstration case.

With the same approach, we obtain the width of the vehicle $w_{\mathrm{V}}$ by finding the length of the regions that contain the most nonnull couriers at the first column $(n=0)$, as shown in Fig. 13(b), with dark gray squares, and we define the width of vehicle $w_{\mathrm{V}}$ starting from $m=8$ to $m=M-1$ in this demonstration case.

The second procedure is to find the location of key feature courier $K_{1}$. As the width of the roof should be smaller or equals the width of vehicle $w_{\mathrm{V}}$, we therefore define the location of the key feature courier $K_{1}$ to be within the region that is $N-l_{r} \leq$ $n \leq N-1$ and $0 \leq m \leq w_{\mathrm{V}}-1$. In the demonstration case, the searching region of $K_{1}$ is $12 \leq n \leq 15$ and $0 \leq m \leq 7$, as shown as dashed square lines in Fig. 14(a).

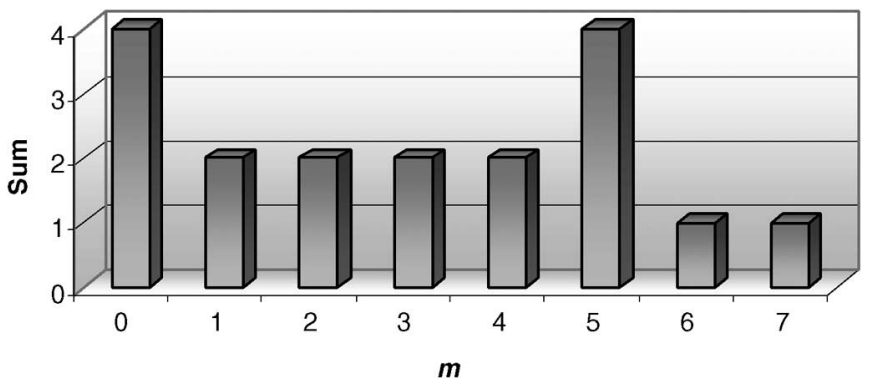

Fig. 15. Location of $K_{1}$.

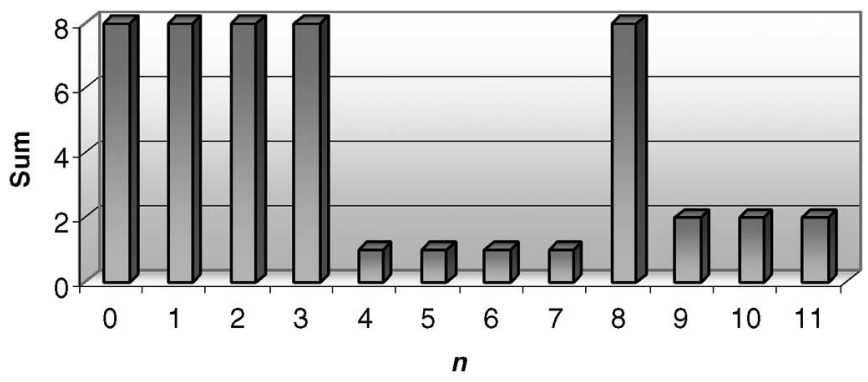

Fig. 16. Location of $K_{2}$ and $K_{3}$.

As $K_{1}$ contains the highest value of textual couriers toward the vanishing point $\mathrm{VP}_{X}$, we can therefore sum up the horizontal value of textual couriers of every row within the searching region of $K_{1}$ and find the location that contains the highest value, as shown in Fig. 15, in this demonstration. We can see that the highest value is located when $m=5$, whereas the contour of the vehicle is located on $m=0$. Subsequently, we identify the location of key feature courier $K_{1}=(5,12)$ in this demonstration case.

The third procedure is to find the locations of key feature couriers $K_{2}$ and $K_{3}$. As we know the width of vehicle $w_{\mathrm{V}}$ and the location of key feature courier $K_{1}$, therefore, we can define the locations of key feature couriers $K_{2}$ and $K_{3}$ to be within the searching region $0 \leq n<N-l_{\mathrm{r}}$ and $\mathrm{NC}_{m} \leq$ $m<\mathrm{NC}_{m}+w_{\mathrm{V}}$, where $\mathrm{NC}_{m}$ is the number of null couriers of each column at the top. In the demonstration case, the searching region of $K_{1}$ is $0 \leq n<12$ and $\{8, \ldots, 1\} \leq$ $m \leq\{15, \ldots, 8\}$, as shown as dashed square lines in Fig. 14(b).

As the region between $K_{2}$ and $K_{3}$ contains the lowest textual couriers, we can therefore sum up the value of vertical textual couriers of every column within the searching region and find the longest locations that have a lower value, which means that the value is lower than the median of those values. In this demonstration case, we can see that the longest locations that contain relatively low values are from $n=4$ to $n=7$, as shown in Fig. 16, and therefore, we can define the boundaries at $n=3$ and $n=8$. Consequently, we identify the locations of key feature couriers $K_{2}=$ $(9,8)$ and $K_{3}=(10,3)$ in this demonstration case, where there are three and two null couriers at $n=3$ and $n=8$, respectively.

Once we get these three key feature couriers, we can inverse transform the corresponding pixel of input frame $f_{\mathrm{i}}$ from each key feature courier, and the pixel location of each key feature 


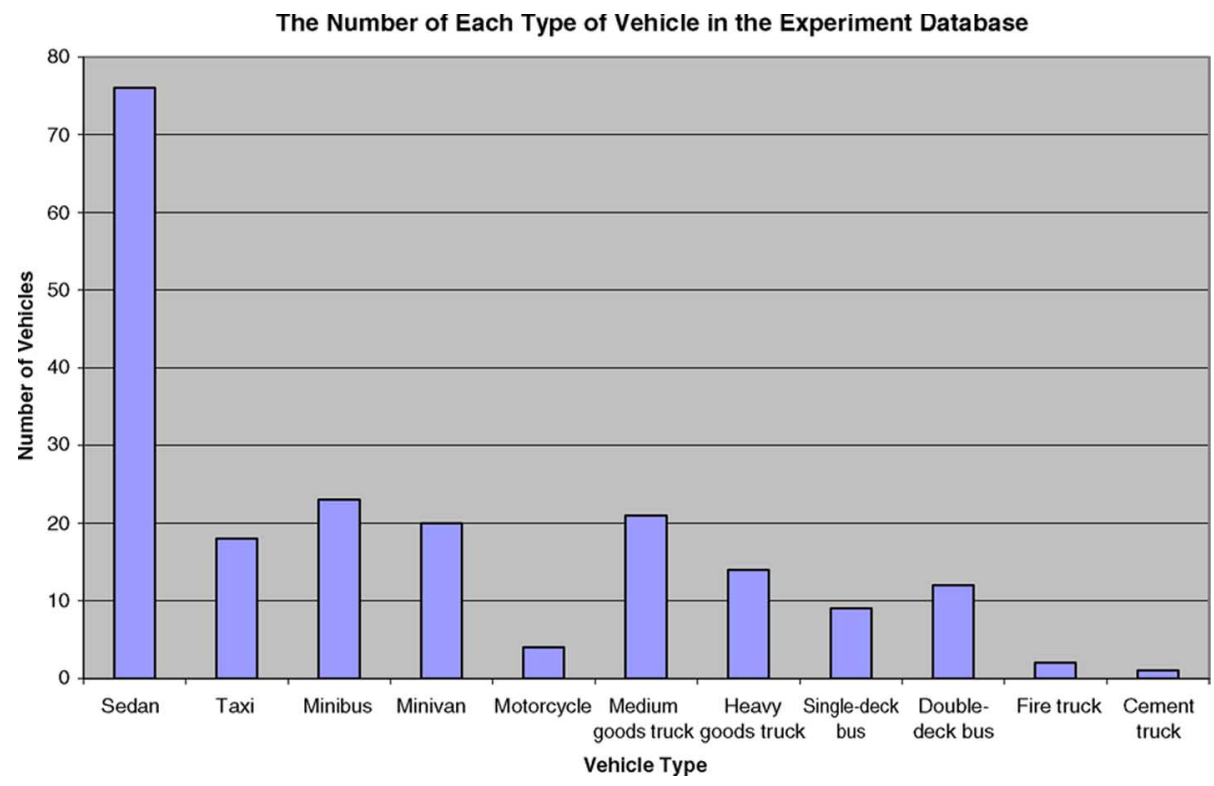

Fig. 17. Number of each type of vehicle in the experiment database.

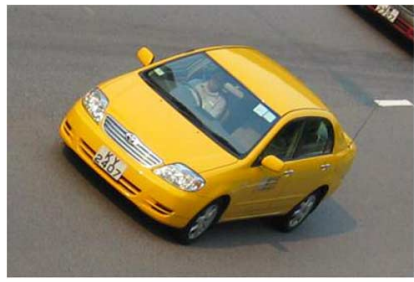

(a)

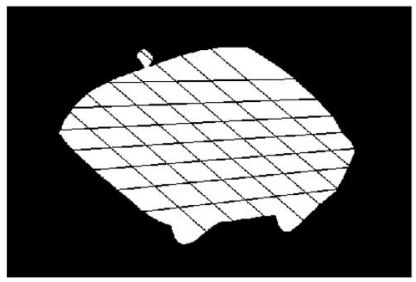

(b)
Fig. 18. Partitions on a yellow sedan. (a) Input frame $f_{\mathrm{i}}$. (b) Scale 3 regions.

point of the input image can be calculated by finding the central pixel of those corresponding pixels as

$$
x_{u}=\frac{\sum_{i, j \in K_{u}} i \cdot O_{\mathrm{V}}(i, j)}{\sum_{i, j \in K_{u}} O_{\mathrm{V}}(i, j)} \quad y_{u}=\frac{\sum_{i, j \in K_{u}} j \cdot O_{\mathrm{V}}(i, j)}{\sum_{i, j \in K_{u}} O_{\mathrm{V}}(i, j)}
$$

where $u \in 1,2,3$, and $x_{u}$ and $y_{u}$ are the central pixels of those key feature points $K_{u}$. Afterward, we can segment those component regions, as shown in Fig. 7, to segment those vehicle components.

\section{RESUlTS AND Discussions}

The proposed method was evaluated on 200 sets of realworld monocular traffic image sequences that have been captured under different viewing angles, vehicle sizes, and colors for testing. All the images were taken in an outdoor environment where the effects of shadow and reflection of vehicle chassis are prominent. The set of images contains most of the vehicle types on the road, including sedan, taxi, minibus, minivan, motorcycle, medium goods truck, heavy goods truck, single-deck bus, double-deck bus, and special vehicles such as fire and cement trucks. The number of each type of vehicle is shown in Fig. 17.

For evaluation purposes, the reference key feature points $K_{1}^{\prime}$, $K_{2}^{\prime}$, and $K_{3}^{\prime}$ of vehicle are required for subsequent calculation of classification error. These key feature points $K_{1}^{\prime}, K_{2}^{\prime}$, and $K_{3}^{\prime}$
TABLE III

CRITICAL SCALE LEVEL

\begin{tabular}{|c|c|c|c|c|}
\hline $\boldsymbol{L}$ & $\boldsymbol{T}^{L}$ & $(\boldsymbol{m}+\mathbf{1})(\boldsymbol{n}+\mathbf{1})$ & $\boldsymbol{S}^{L}$ & $\boldsymbol{S}^{L+1}-\boldsymbol{S}^{L}$ \\
\hline 0 & 0 & 1 & $0.00 \%$ & $0.00 \%$ \\
\hline 1 & 0 & 4 & $0.00 \%$ & $0.00 \%$ \\
\hline 2 & 0 & 16 & $0.00 \%$ & $0.00 \%$ \\
\hline 3 & 0 & 64 & $0.00 \%$ & $0.00 \%$ \\
\hline 4 & 0 & 256 & $0.00 \%$ & $0.00 \%$ \\
\hline 5 & 0 & 1,024 & $0.00 \%$ & $0.12 \%$ \\
\hline 6 & 5 & 4,096 & $0.12 \%$ & $0.10 \%$ \\
\hline 7 & 37 & 16,384 & $0.23 \%$ & $27.01 \%$ \\
\hline 8 & 17,849 & 65,536 & $27.24 \%$ & $4.06 \%$ \\
\hline 9 & 82,045 & 262,144 & $31.03 \%$ & N. A. \\
\hline \multicolumn{5}{|r}{}
\end{tabular}

are manually defined by combining the visual observation on images and the human knowledge about the vehicle. The error rate ER of each component region $R$ is calculated according to the number of pixels of the error difference region $R_{\mathrm{d}}$ and the number of pixels of the reference region $R_{\mathrm{f}}$ as

$$
\mathrm{ER}=\frac{\sum_{i, j \in R}\left|R_{\mathrm{d}}(i, j)\right|}{\sum_{i, j \in R} R_{\mathrm{f}}(i, j)}
$$

where $R_{\mathrm{d}}$ indicates the difference between the reference region $R_{\mathrm{f}}$ and the computed region $R_{\mathrm{V}}$ as

$$
R_{\mathrm{d}}(i, j)=R_{\mathrm{f}}(i, j)-R_{\mathrm{V}}(i, j) .
$$

Out of all the tested images, a yellow sedan sample, as shown in Fig. 18(a), was chosen to illustrate in details the working of the proposed method, and the corresponding scale 3 regions $R^{3}$ are shown in Fig. 18(b). We can see that regions $R_{0,0}^{3}$, $R_{0,1}^{3}, R_{1,0}^{3}, R_{6,7}^{3}, R_{7,6}^{3}$, and $R_{7,7}^{3}$ are not within the vehicle mask, and therefore, their corresponding textual couriers $R_{T 0,0}^{3}$, $R_{T 0,1}^{3}, R_{T 1,0}^{3}, R_{T 6,7}^{3}, R_{T 7,6}^{3}$, and $R_{T 7,7}^{3}$ are to be defined as null couriers.

After constructing the multiscale textural couriers $R_{T}^{L}$, we compute the ratio $S L$ of each scale level, as presented in 


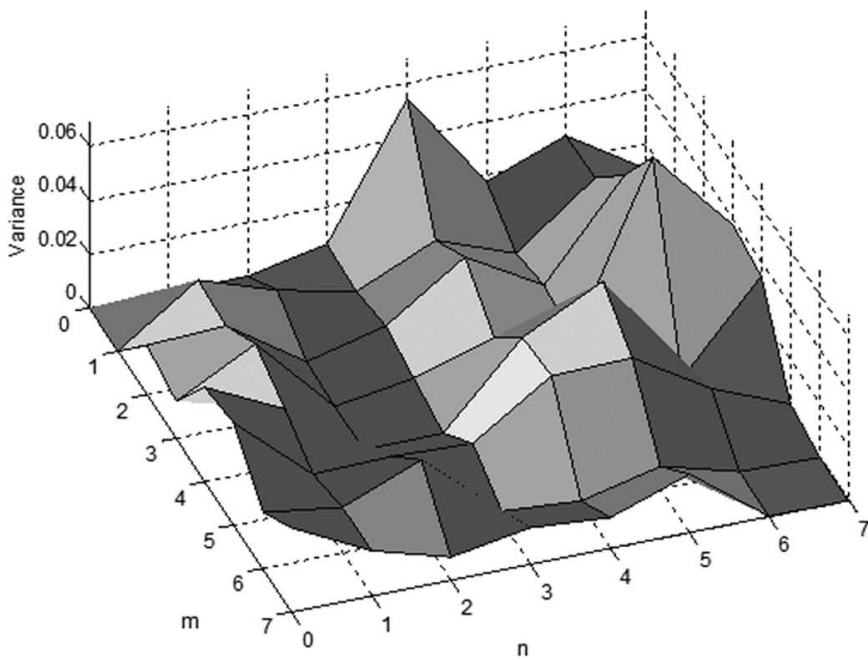

(a)

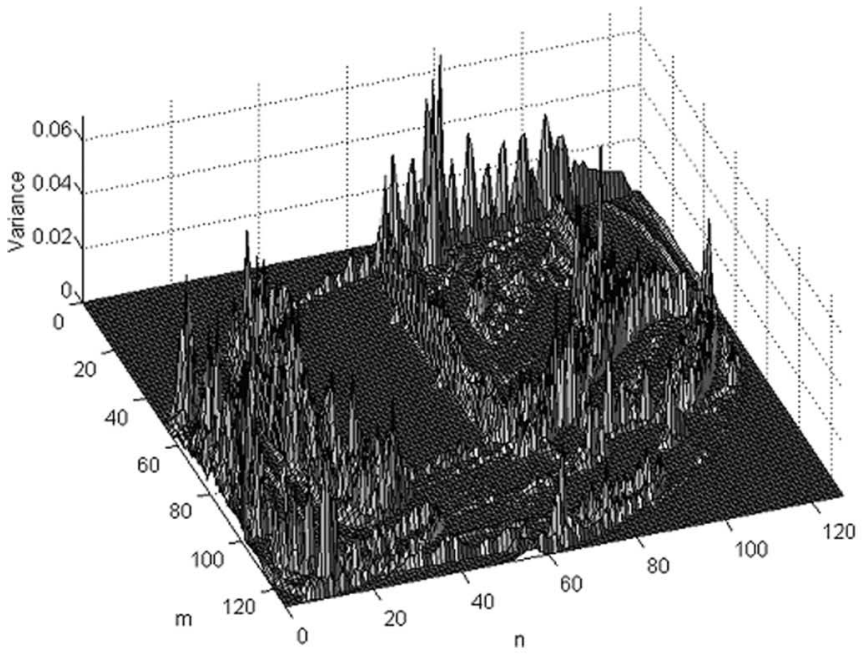

(c)

Fig. 19. Texture couriers $R_{T}^{L}$. (a) $L=3$. (b) $L=6$. (c) $L=7$. (d) $L=8$.

Table III. We can see that $C=7$ as $\max \left(S^{L+1}-S^{L}\right)$ occurs on scale level 7.

With reference to Table III, the critical textural scale $C$ occurs at scale 7. To show the trend of courier surfaces, Fig. 19 shows the surfaces of textural couriers $R_{T}$ in 3-D at scales 3, 6,7 , and 8 . As can be seen, scale 3 is too coarse to enable a good segmentation, whereas scale 8 contains too much detail. If a scale lower than $C$ is chosen, the component characteristics are insufficient to warrant an accurate identification. On the other hand, if a scale higher than $C$ is instead chosen, too many superfluous features have to be computed and discriminated.

With the textural-courier map $T(m, n)$, we are able to get the key feature couriers $K_{1}, K_{2}$, and $K_{3}$ based on their topological structure. We start to determine the length of the roof $l_{\mathrm{r}}$ by finding the length of the regions that contain the most nonnull couriers at the first row. Fig. 20 shows the total sum of top-left null couriers of each column that projected into the first row. The values of locations $105 \leq n \leq 126$ are zero at the first row, and therefore, the edge between the roof and the windscreen occurs on $n=105$. Curiously, the values of location $80 \leq n \leq 83$ are also zero, which indicates the region of the side mirror.

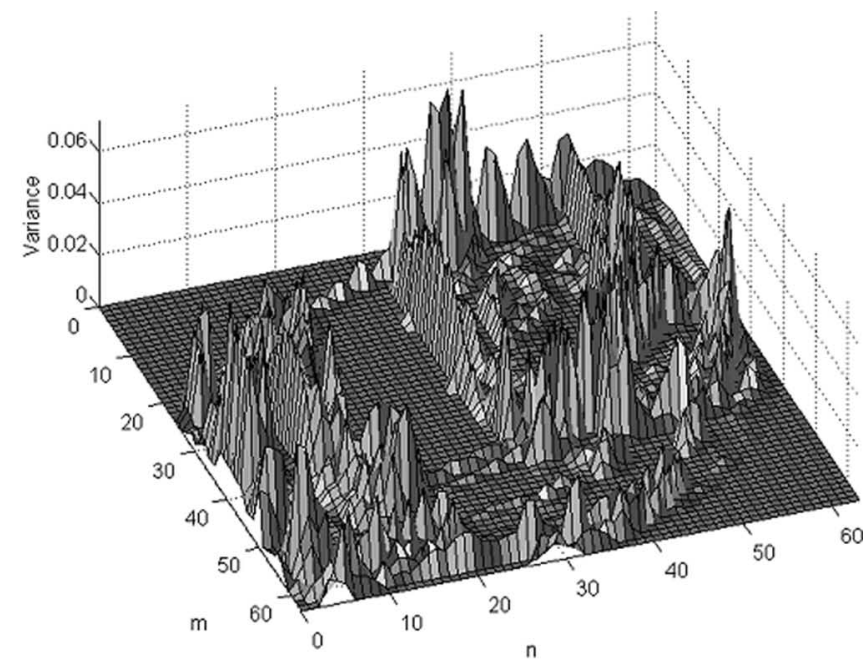

(b)

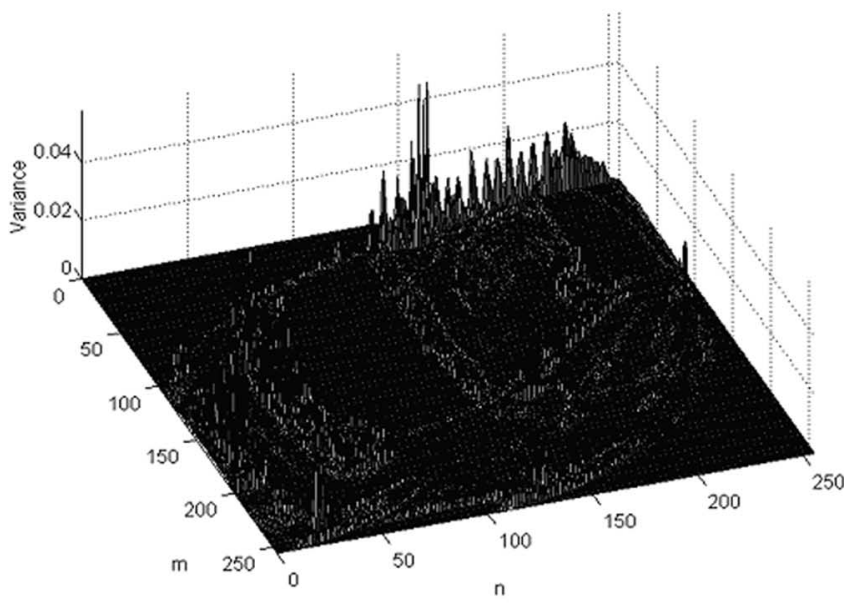

(d)

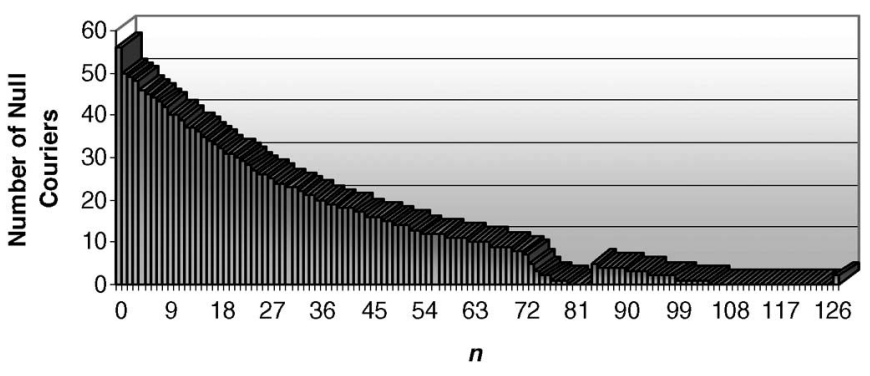

Fig. 20. Length of roof.

We then determine the width of the vehicle $w_{\mathrm{V}}$ by finding the length of the regions that contain the most nonnull couriers at the first row. Fig. 21 shows the total sum of the top-left null couriers of each row that projected into the first column. The values of locations $56 \leq n \leq 115$ at the first row are zero, and therefore, we can start counting the width of the vehicle $w_{\mathrm{V}}$ from $n=56$ to $n=127$.

As $K_{1}$ contains the highest value of textual couriers toward the vanishing point $\mathrm{VP}_{X}$, we therefore sum up the horizontal textual-courier values of every row within the searching region 


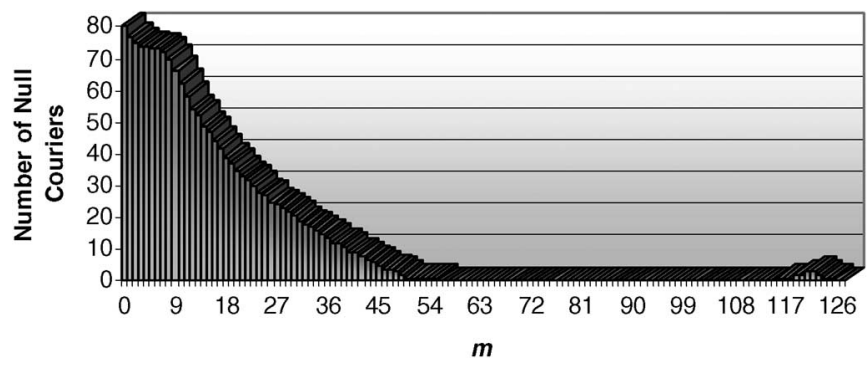

Fig. 21. Width of vehicle.

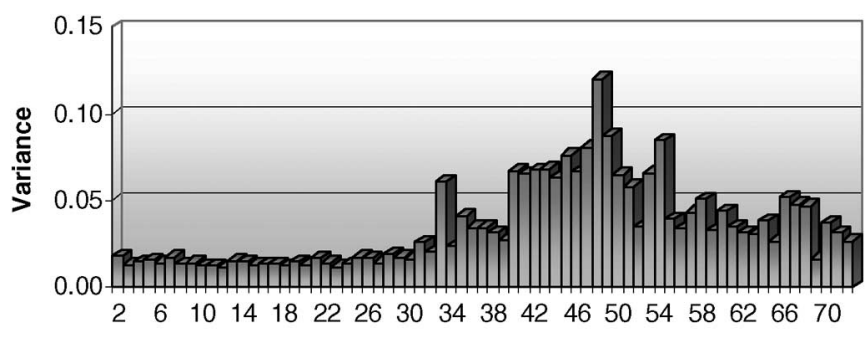

Fig. 22. Location of $K_{1}$.

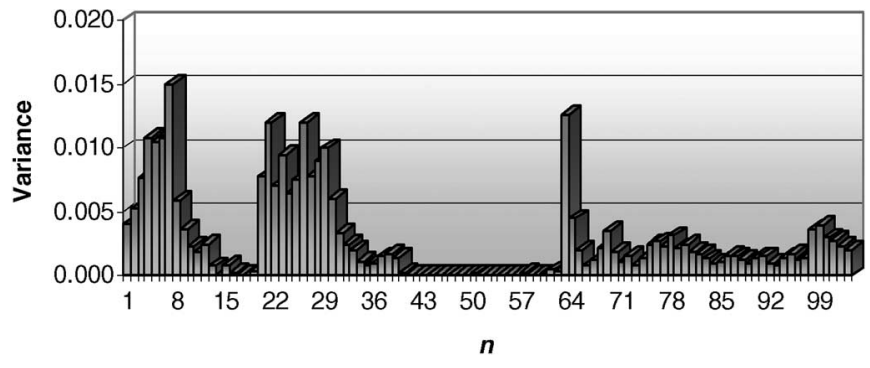

Fig. 23. Location of $K_{2}$ and $K_{3}$.

of $K_{1}$ and find the location that contains the highest value, as shown in Fig. 22. We can see that the highest value is located when $m=48$, and therefore, we can estimate the location of key feature courier $K_{1}=(48,105)$ in this sample.

As the region between $K_{2}$ and $K_{3}$ contains the lowest textual couriers, we can therefore sum up the vertical value of textual couriers of every column within the searching region and find the longest locations that have a lower value. In this sample, the median value is about 0.00151 , and we have found that the longest locations that contain relatively low values are from $n=39$ to $n=62$, as shown in Fig. 23; thus, we can estimate the location of key feature couriers $K_{2}=(92,38)$ and $K_{3}=(83,63)$, where there are 21 and 12 null couriers at $n=38$ and $n=63$, respectively.

Once we have computed these three key feature couriers, we can inverse transform the corresponding pixels of input frame $f_{\mathrm{i}}$ from each key feature courier. The pixel location of key feature points are shown in Fig. 24(a), and the component boundaries are shown in Fig. 24(b). For comparison, the component boundaries with the reference key feature points $K_{1}^{\prime}, K_{2}^{\prime}$, and $K_{3}^{\prime}$ that are manually defined are shown in Fig. 25.

The corresponding component regions $R_{\mathrm{R}}, R_{\mathrm{W}}, R_{\mathrm{B}}, R_{\mathrm{G}}$, $R_{\mathrm{S}}$, and $R_{\mathrm{D}}$ are identified, as shown in Fig. 26(a)-(f), respectively. The black region indicates that the algorithm mistakenly identifies the other regions as part of the local region, whereas the white region conversely indicates that the algorithm identi-

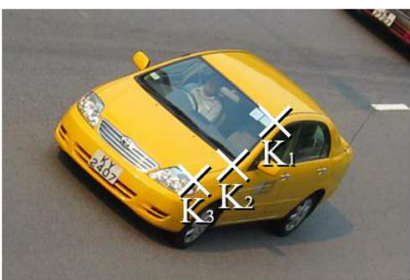

(a)

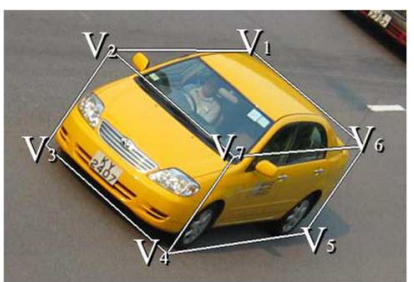

(b)
Fig. 24. Yellow sedan. (a) Key feature points. (b) Segmented regions.

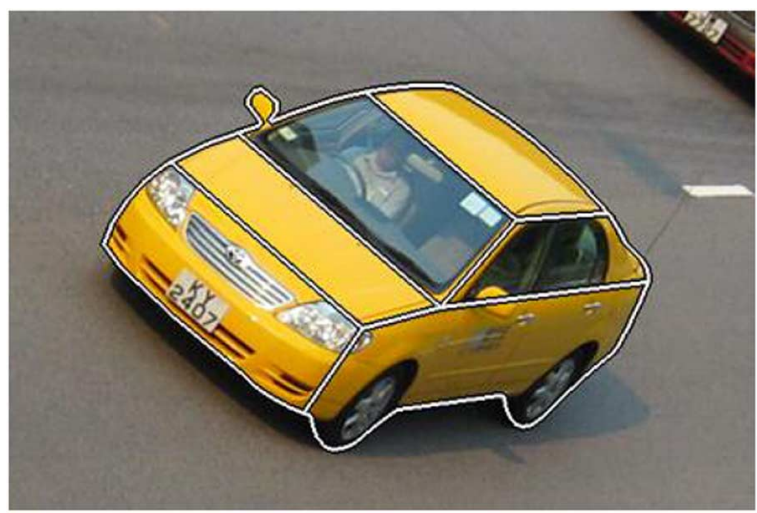

Fig. 25. Reference component regions.



(a)

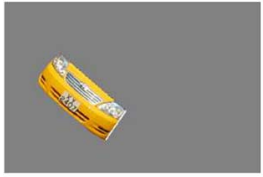

(d)

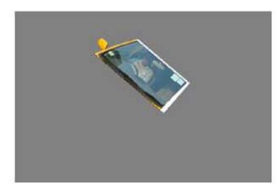

(b)

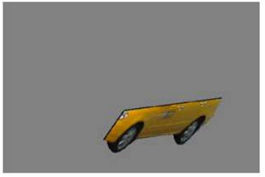

(e)

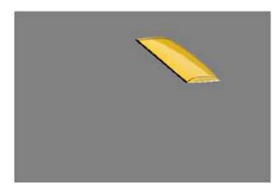

(c)

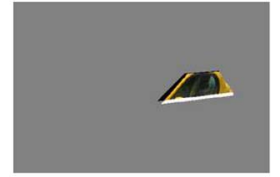

(f)
Fig. 26. Vehicle components. (a) $R_{\mathrm{B}}$. (b) $R_{\mathrm{W}}$. (c) $R_{\mathrm{R}}$. (d) $R_{\mathrm{G}}$. (e) $R_{\mathrm{D}}$. (f) $R_{\mathrm{S}}$.

fies itself as the others. The ER of regions $R_{\mathrm{R}}, R_{\mathrm{W}}, R_{\mathrm{B}}, R_{\mathrm{G}}$, $R_{\mathrm{S}}$, and $R_{\mathrm{D}}$ are found to be $7.6 \%, 8.2 \%, 4.6 \%, 1.4 \%, 17.3 \%$, and $3.9 \%$, respectively. Fig. 26(c) shows the capable result of segmenting roof, although there is a reflection boundary on the roof. This error would not significantly affect vehicle classification, as vehicle components (i.e., roof, windscreen, bonnet, grille, side windows, and door panels) were successfully identified, despite the presence of slight segmentation error due to minor variations in vehicle geometry. As such, there is a sufficient information to construct a relational graph that represents the vehicle for classification by means of graph matching.

The proposed method also works well with the vehicle that has a roof, as shown in Fig. 27(a). Fig. 27(b) shows the surfaces of textural courier $R_{T}^{C}$ in 3-D at critical scale 7 . With the same approach, we inverse transform the corresponding pixels of input frame $f_{\mathrm{i}}$ from each key feature courier. The pixel locations of key feature points are shown in Fig. 28(a), and the component boundaries are shown in Fig. 28(b). 


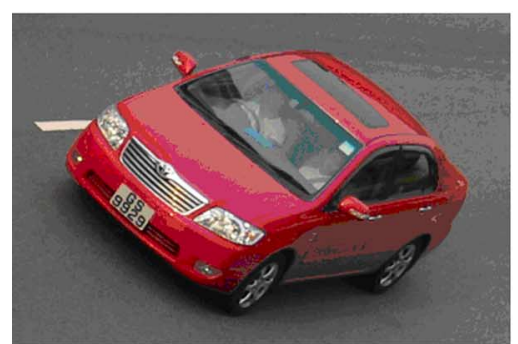

(a)

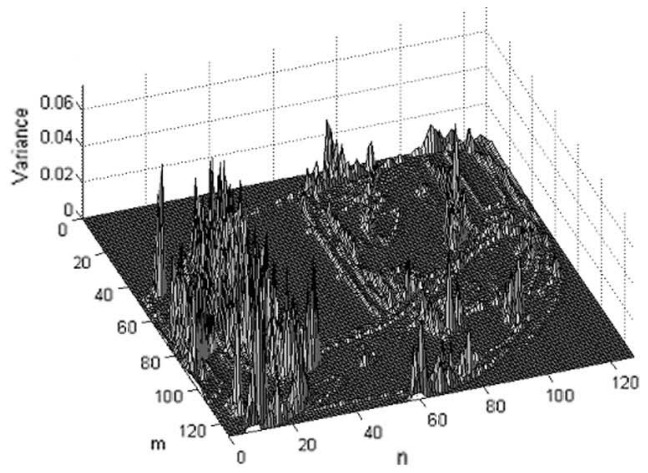

(b)

Fig. 27. Red sedan. (a) Input frame $f_{\mathrm{i}}$. (b) Texture couriers $R_{T}^{C}$.

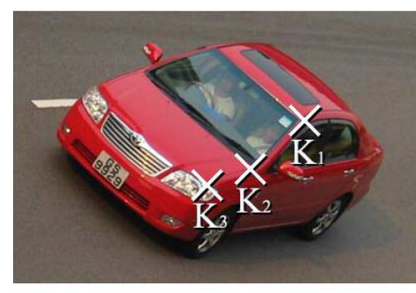

(a)

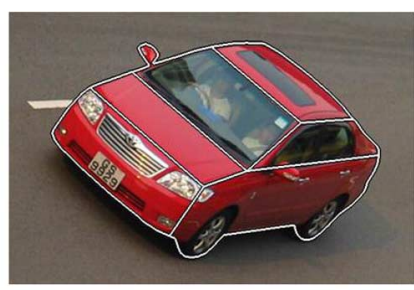

(b)

Fig. 28. Red sedan. (a) Key feature points. (b) Segmented regions.

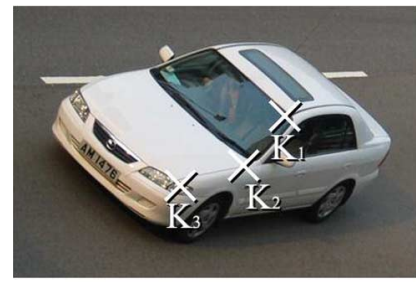

(a)

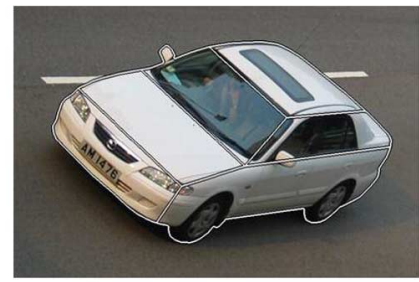

(b)

Fig. 29. White Sedan. (a) Key feature points. (b) Segmented regions.

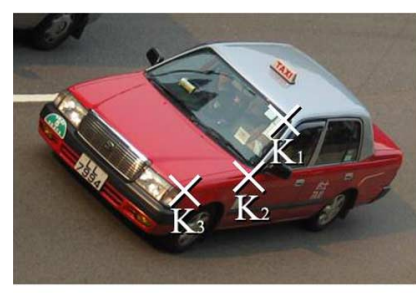

(a)

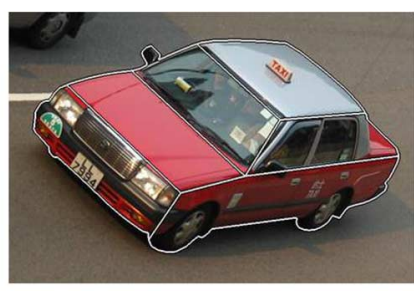

(b)

Fig. 30. Red taxi. (a) Key feature points. (b) Segmented regions.

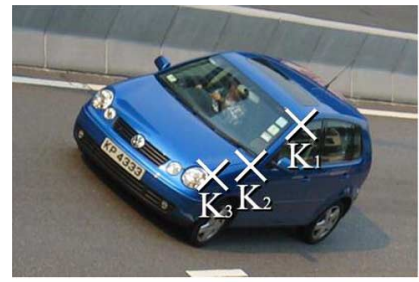

(a)



(b)

Fig. 31. Blue hatchback. (a) Key feature points. (b) Segmented regions.

In the third sample, a white sedan with a roof window was tested, and the result of the estimated key feature points is shown in Fig. 29(a). This vehicle can be segmented into the

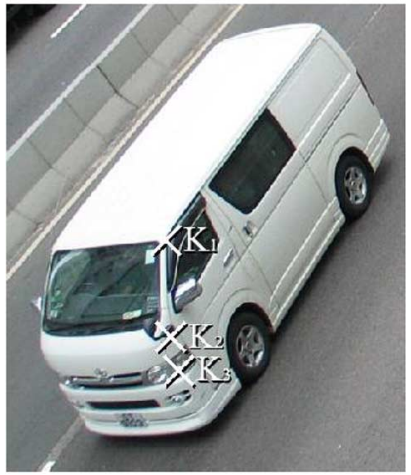

(a)

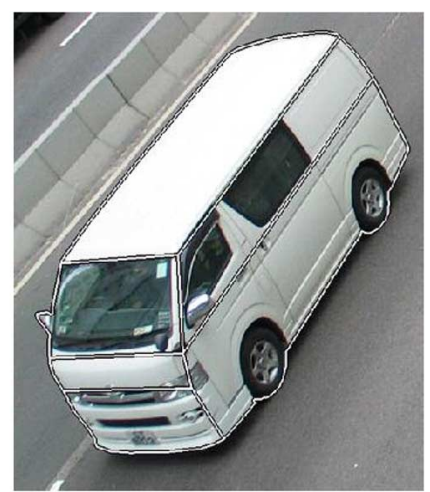

(b)
Fig. 32. White van. (a) Key feature points. (b) Segmented regions.

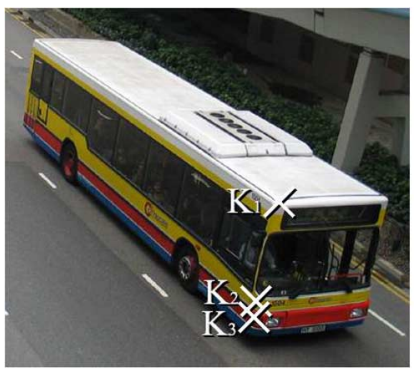

(a)

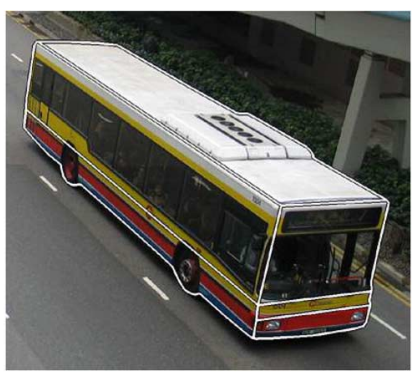

(b)
Fig. 33. Yellow bus. (a) Key feature points. (b) Segmented regions.

predefined component regions, as shown in Fig. 29(b), except the edge between $K_{1}$ and $K_{2}$ that cannot be located on the boundary between the windscreen and side windows. In the fourth sample, a red taxi with a light box cap was tested, and the results of the estimated key feature points and the identified component regions are shown in Fig. 30(a) and (b), respectively. Although the roof contains reflection regions and light box cap, the result of the segmented component regions is promising. In the fifth sample, a blue hatchback with a roof window was tested, and the result of the estimated key feature points is shown in Fig. 31(a). The boundaries of the segmented component regions are highly qualified with this streamlined type vehicle, as shown in Fig. 31(b). In the sixth sample, a white van was tested, and the result of the estimated key feature points is shown in Fig. 32(a). This vehicle can be segmented into the 


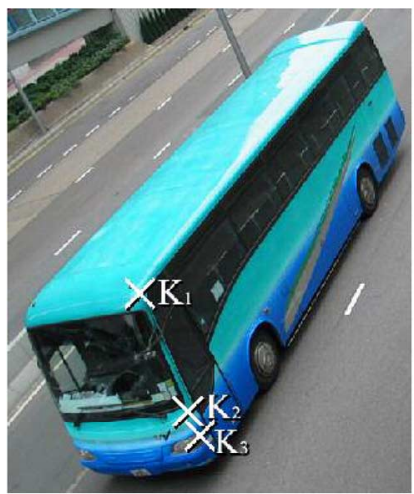

(a)



(b)
Fig. 34. Blue bus. (a) Key feature points. (b) Segmented regions.

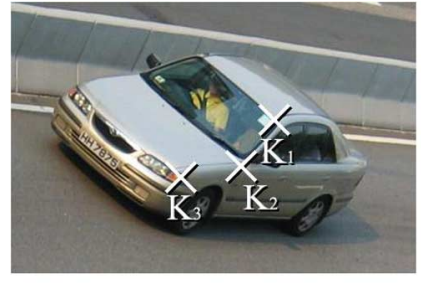

(a)

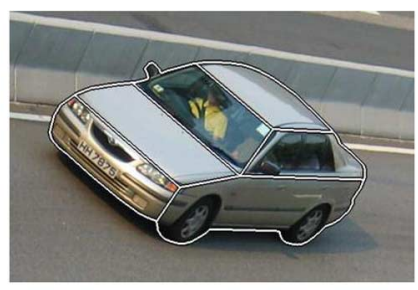

(b)
Fig. 35. Silver sedan. (a) Key feature points. (b) Segmented regions.

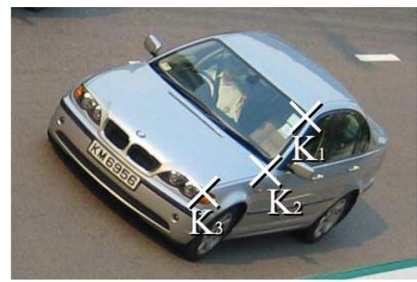

(a)

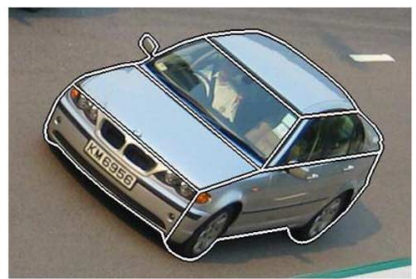

(b)
Fig. 36. Silver sedan. (a) Key feature points. (b) Segmented regions.

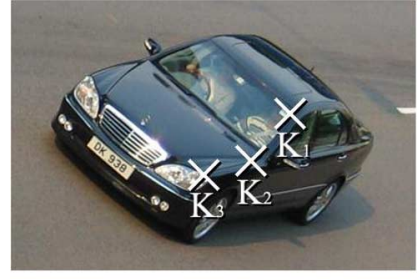

(a)

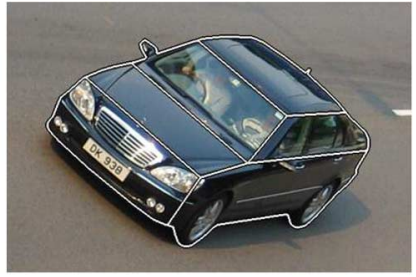

(b)
Fig. 37. Black sedan. (a) Key feature points. (b) Segmented regions.

predefined component regions, as shown in Fig. 32(b), and the result is very promising.

For large-sized vehicles, the key feature point can also be determined. In the seventh sample, a yellow bus was tested, and the results of the estimated key feature points and the identified component regions are shown in Fig. 33(a) and (b), respectively. In the eighth sample, a blue bus of different model type was tested, and the results of the estimated key feature points and the identified component regions are shown in Fig. 34(a) and (b), respectively.

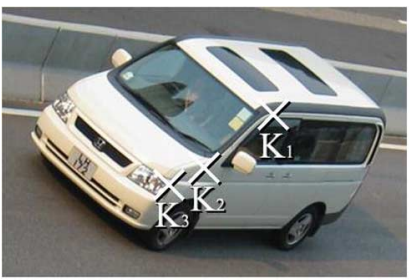

(a)

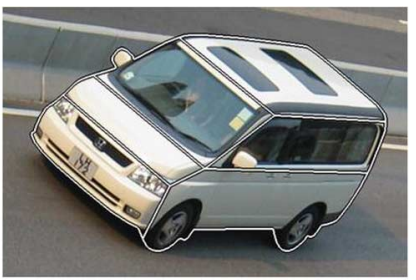

(b)
Fig. 38. White van. (a) Key feature points. (b) Segmented regions.

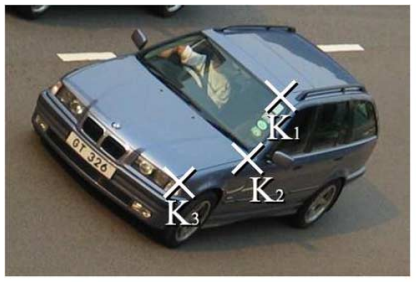

(a)

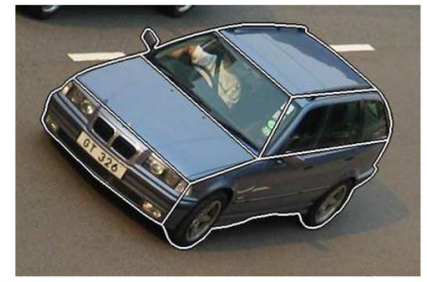

(b)
Fig. 39. Silver blue wagon. (a) Key feature points. (b) Segmented regions.

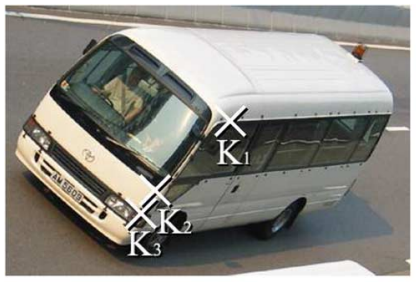

(a)

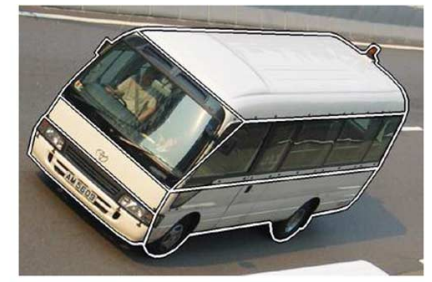

(b)
Fig. 40. White minibus. (a) Key feature points. (b) Segmented regions.

Generally, the silver and black streamlined vehicles are the worst scenario, and an ill-defined problem in typical research studies (as this scenario) does not provide any chrominance features; in addition, the luminance values of the vehicles are lower than the road surface. Advantageously, this scenario can be solved well with textual properties, as demonstrated in the following three sample cases. In the ninth sample, a silver sedan was tested, and the results of the estimated key feature points and the identified component regions are shown in Fig. 35(a) and (b), respectively. In the tenth sample, a silver sedan of different model type was tested, and the results of the estimated key feature points and the identified component regions are shown in Fig. 36(a) and (b), respectively. In the 11 th sample, it is also the hardest case because the luminance values are relatively low in most regions. A black sedan with a roof window was tested, and the results of the estimated key feature points and the identified component regions are shown in Fig. 37(a) and (b), respectively.

In the 12th sample, a white van with roof windows was tested; the results of the estimated key feature points and the identified component regions are shown in Fig. 38(a) and (b), respectively. In the 13th sample, a silver blue wagon was tested, and the results of the estimated key feature points and the identified component regions are shown in Fig. 39(a) and (b), respectively. However, the edge between the roof and side windows cannot properly be computed because it locates on the black handle on the roof. In the 14th sample, the results of 


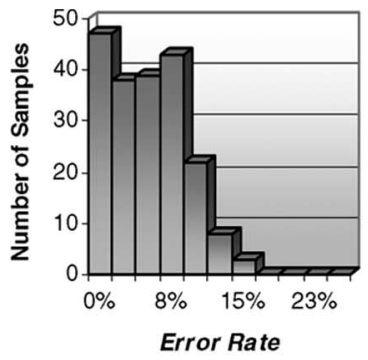

(a)

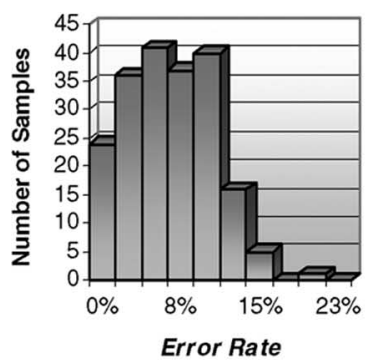

(d)

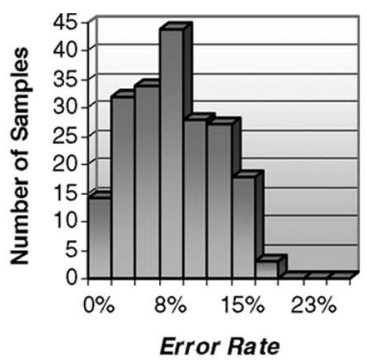

(b)

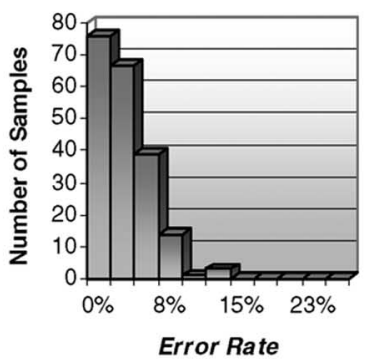

(e)

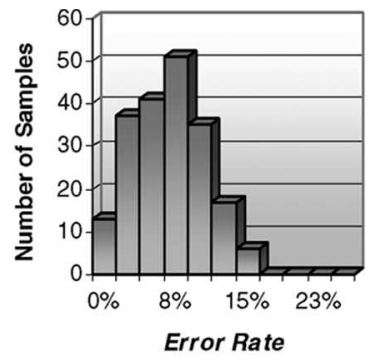

(c)

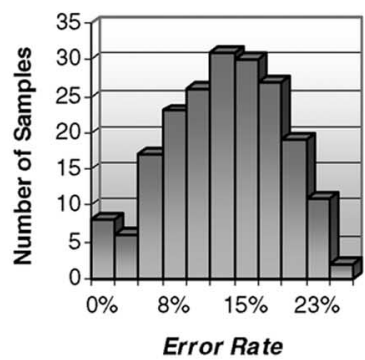

(f)

Fig. 41. Error rates of individual component region. (a) $R_{\mathrm{B}}$. (b) $R_{\mathrm{W}}$. (c) $R_{\mathrm{R}}$. (d) $R_{\mathrm{G}}$. (e) $R_{\mathrm{D}}$. (f) $R_{\mathrm{S}}$.

TABLE IV

Computational-Time Requirement of the Proposed Method

\begin{tabular}{|c|c|}
\hline Proposed step & Computational time \\
\hline Vehicle component identification & $0.42 \mathrm{sec}$ \\
\hline Multi-scale texture couriers construction & $0.68 \mathrm{sec}$ \\
\hline Key feature points estimation & $0.19 \mathrm{sec}$ \\
\hline Overall & $1.29 \mathrm{sec}$ \\
\hline
\end{tabular}

the estimated key feature points and the identified component regions are shown in Fig. 40(a) and (b), respectively. The edge between the roof and side windows does not properly locate, but the edge of large vehicle can be estimated by using the 3-D cuboid model to calculate the length between vertices $V_{6}$ and $V_{7}$, as mentioned in [9].

Finally, the error rates of all 200 cases based on the number of pixels of the reference component regions are statistically shown in Fig. 41. The error rates of components of regions $R_{\mathrm{R}}, R_{\mathrm{W}}, R_{\mathrm{B}}, R_{\mathrm{G}}, R_{\mathrm{S}}$, and $R_{\mathrm{D}}$ are found to be $6.7 \%, 7.6 \%$, $4.9 \%, 6.3 \%, 12.6 \%$, and $2.6 \%$, respectively. The error rates of windscreen and side windows region are the highest as there are some undesired features around the windscreen like the stickers on the windscreen or the water-resistant plastic on side windows, etc., which affect the accuracy of the results.

The computational time of the three major steps of the proposed method is depicted in Table IV. It was implemented in MATLAB on a Pentium III 800-MHz platform, and the computational time of each step was monitored by MATLAB. The multiscale-textural-courier-construction step is the slowest among the three steps, whereas the feature-pointestimation step is the fastest. This is not surprising, as the former involves the decomposition of vehicle mask into a multiscale region in order to generate textural couriers, which is quite time-consuming. However, this delay is acceptable, as the overall computational time is only slightly more than 1 s/image. As such, the proposed method is computationally feasible.

\section{FUTURE DEVELOPMENTS}

For the proposed methodology, the component regions are identified by segmenting the boundaries based on camera parameters and vanishing points. The exact contour of an individual component cannot be segmented for classification. For instance, the bonnet of most vehicles may not be square, and therefore, one of the possible solution is to track the gradient features of the individual component region to outline the exact contour.

The other possible development is to recover the 3-D information of a vehicle from the displacements of the key feature points between consecutive images. By utilizing the property that these key feature points at different heights on the same vehicle would differently displace in the image, the 3-D coordinates of these feature points can be estimated using their motion vectors. As a result, this 3-D vehicle shape can provide imperative information for analyzing traffic scenes.

\section{CONCLUSION}

In this paper, we have presented a novel scale-space method for identifying vehicle components. In the proposed method, the key feature points of the vehicle have been described as symbolic features in streamlined vehicles; therefore, the proposed method is fundamentally different from the majority of existing model-based algorithms. Based on the topological structure of multiscale textural couriers, we are able to determine the regions that are homogenous in texture from which vehicle components can be identified. A set of algorithms has been described and compared in this paper to locate the symbolic features. Successful results have been obtained with a variety of traffic scenes and streamlined vehicles. The use of a priori knowledge about the scene and the objects has been shown to be the key to the success of the novel algorithms. Used in conjunction with the textural difference, vehicle components can be differentiated. With this vehicle-component 
representation in hand, further identification of vehicles can be made. The current paper has demonstrated the efficiency and robustness of context-based computer vision.

\section{REFERENCES}

[1] R. P. Avery, Y. Wang, and G. S. Rutherford, "Length-based vehicle classification using images from uncalibrated video cameras," in Proc. IEEE Conf. Intell. Transp. Syst., Oct. 2004, pp. 737-742.

[2] C. J. Chen, C. C. Chiu, B. F. Wu, S. P. Lin, and C. D. Huang, "The moving object segmentation approach to vehicle extraction," in Proc. IEEE Int. Conf. Netw., Sens. Control, Mar. 2004, vol. 1, pp. 19-23.

[3] J. M. Collado, C. Hilario, A. de la Escalera, and J. M. Armingol, "Model based vehicle detection for intelligent vehicles," in Proc. IEEE Intell. Veh. Symp., Jun. 2004, pp. 572-577.

[4] G. S. K. Fung, N. H. C. Yung, and G. K. H. Pang, "Vehicle shape approximation from motion for visual traffic surveillance," in Proc. IEEE Conf. Intell. Transp. Syst., Aug. 2001, pp. 608-613.

[5] G. S. K. Fung, N. H. C. Yung, G. K. H. Pang, and A. H. S. Lai, "Camera calibration from road lane markings," Opt. Eng., vol. 42, no. 10, pp. 29672977, Oct. 2003.

[6] S. Gupte, O. Masoud, and N. P. Papanikolopoulos, "Vision-based vehicle classification," in Proc. IEEE Conf. Intell. Transp. Syst., Oct. 2000, pp. 46-51.

[7] S. Kamijo and M. Sakauchi, "Segmentation of vehicles and pedestrians in traffic scene by spatio-temporal Markov random field model," in Proc. IEEE Int. Conf. Acoust., Speech, Signal Process., Apr. 2003, vol. 3, pp. 361-364.

[8] N. K. Kanhere, S. J. Pundlik, and S. T. Birchfield, "Vehicle segmentation and tracking from a low-angle off-axis camera," in Proc. IEEE Comput. Soc. Conf. Comput. Vis. Pattern Recog., Jun. 2005, vol. 2, pp. 1152-1157.

[9] A. H. S. Lai, G. S. K. Fung, and N. H. C. Yung, "Vehicle type classification from visual-based dimension estimation," in Proc. IEEE Conf. Intell. Transp. Syst., Aug. 2001, pp. 203-208.

[10] A. H. S. Lai and N. H. C. Yung, "A fast and accurate scoreboard algorithm for estimating stationary backgrounds in an image sequence," in Proc. IEEE Int. Symp. Circuits Syst., May 1998, vol. 4, pp. 241-244.

[11] W. W. L. Lam, C. C. C. Pang, and N. H. C. Yung, "A highly accurate texture-based vehicle segmentation method," Opt. Eng., vol. 43, no. 3, pp. 591-603, Mar. 2004.

[12] W. W. L. Lam, C. C. C. Pang, and N. H. C. Yung, "Multi-scale space vehicle component identification," in Proc. IEEE Int. Conf. Image Process., Oct. 2004, vol. 2, pp. 925-928.

[13] P. Li, L. Ding, and J. Liu, "A video-based traffic information extraction system," in Proc. IEEE Intell. Veh. Symp., Jun. 2003, pp. 528-532.

[14] C. C. C. Pang, W. W. L. Lam, and N. H. C. Yung, "A novel method for resolving vehicle occlusion in a monocular traffic image sequence," IEEE Trans. Intell. Transp. Syst., vol. 5, no. 3, pp. 129-141, Sep. 2004.

[15] S. Pumrin and D. J. Dailey, "Vehicle image classification via expectation-maximization algorithm," in Proc. Int. Symp. Circuits Syst., May 2003, vol. 2, pp. 468-471.

[16] O. Sidla, L. Paletta, Y. Lypetskyy, and C. Janner, "Vehicle recognition for highway lane survey," in Proc. IEEE Conf. Intell. Transp. Syst., Oct. 2004, pp. 531-536.

[17] J. C. Tai and K. T. Song, "Background segmentation and its application to traffic monitoring using modified histogram," in Proc. IEEE Int. Conf. Netw., Sens. Control, Mar. 2004, vol. 1, pp. 13-18.

[18] T. N. Tan and K. D. Baker, "Efficient image gradient based vehicle localization," IEEE Trans. Image Process., vol. 9, no. 8, pp. 1343-1356, Aug. 2000.

[19] W. Wei, Q. S. Zhang, and M. Wang, "A method of vehicle classification using models and neural networks," in Proc. IEEE Conf. Veh. Technol., May 2001, pp. 3022-3026.

[20] L. Xie, G. Zhu, Y. Wang, H. Xu, and Z. Zhang, "Robust vehicles extraction in a video-based intelligent transportation systems," in Proc. IEEE Int. Conf. Commun., Circuits Syst., May 2005, vol. 2, p. 890.

[21] S. Xuefeng and R. Nevatia, "A model-based vehicle segmentation method for tracking," in Proc. IEEE Int. Conf. Comput. Vis., Oct. 2005, vol. 2, pp. 1124-1131.

[22] A. Yoneyama, C. H. Yeh, and C. J. Kuo, "Moving cast shadow elimination for robust vehicle extraction based on 2D joint vehicle/shadow models," in Proc. IEEE Conf. Advanced Video Signal Based Surveillance, Jul. 2003, pp. 229-236.

[23] N. H. C. Yung and A. H. S. Lai, "Detection of vehicle occlusion using a generalized deformable model," in Proc. IEEE Int. Symp. Circuits Syst., May 1998, vol. 4, pp. 154-157.
[24] X. Zhuang, "Vehicle detection and segmentation in dynamic traffic image sequences with scale-rate," in Proc. IEEE Conf. Intell. Transp. Syst., Oct. 2004, pp. 570-574.

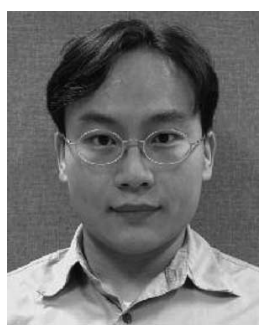

William Wai Leung Lam received the B.Eng. degree in electrical engineering from McMaster University, Hamilton, ON, Canada, and the M.Phil. degree in electrical and electronic engineering from Hong Kong University of Science and Technology, Hong Kong. He is currently working toward the Ph.D. degree with the Laboratory for Intelligent Transportation Systems Research, Department of Electrical and Electronic Engineering, University of Hong Kong.

His research interests include computer vision, intelligent transportation systems, and computer networks.

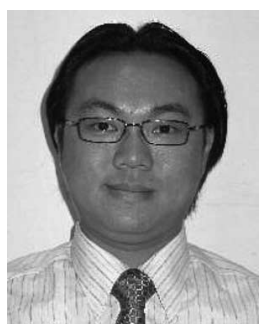

Clement Chun Cheong Pang (S'04-M'07) received the B.Eng. and M.Eng. degrees in electrical and computer engineering from McMaster University, Hamilton, ON, Canada, in 1999 and 2001, respectively, and the $\mathrm{Ph} . \mathrm{D}$. degree in electrical and electronic engineering from the University of Hong Kong (HKU), Hong Kong, in 2005.

He is currently a Senior Research Assistant with the Laboratory for Intelligent Transportation Systems Research, Department of Electrical and Electronic Engineering, HKU, and is currently working toward a unified image algebraic model for outdoor visual traffic surveillance. His research interests include the investigation of statistical models in computer vision, as well as the application of statistical models in railway signaling and communications.



Nelson Hon Ching Yung (S'82-M'85-SM'96) received the B.Sc. and Ph.D. degrees from the University of Newcastle Upon Tyne, Newcastle Upon Tyne, U.K.

From 1985 to 1990 , he was a Lecturer with the University of Newcastle Upon Tyne. From 1990 to 1993 , he was a Senior Research Scientist with the Department of Defence, Canberra, Australia. In late 1993, he joined the University of Hong Kong (HKU), Hong Kong, as an Associate Professor. He leads a research team in digital image processing and intelligent transportation systems. He is the founding Director of the Laboratory for Intelligent Transportation Systems Research, Department of Electrical and Electronic Engineering, HKU, and is also the Deputy Director of HKU's Institute of Transport Studies. He has coauthored five books and book chapters and has published more than 130 journal and conference papers in the areas of digital image processing, parallel algorithms, visual traffic surveillance, autonomous vehicle navigation, and learning algorithms. He is the holder of one U.S. patent and successfully transferred his research to a spinoff technology company. He regularly delivers talks/seminars to government units, professional institutions, associations and commercial companies, and gives interviews to local press regarding his research. He acts as a consultant to a number of local and international companies. He regularly serves as an expert witness for the courts of the HKSAR.

Dr. Yung is a member of the Hong Kong Institution of Engineers and the Institution of Electrical Engineers. He is a Chartered Electrical Engineer. He serves as a Reviewer for the IEEE TRANSACTIONS ON SySTEMS, MAN, AND Cybernetics-PART C, CASVT, Vehicular Technology, Signal Processing, The International Society for Optical Engineers (SPIE) Optical Engineering, SPIE Journal of Electronic Imaging, HKIE Proceedings, Microprocessors and Microsystems, and Robotics and Autonomous Systems Journal. He was a member of the Advisory Panel of the ITS Strategy Review and the Transport Department, HKSAR; the Regional Secretary of the IEEE Asia-Pacific region, a Council Member and the Chairman of Standards Committee of ITS-HK; and the Chair of Computer Division of the International Institute for Critical Infrastructures. He was a Croucher Scholar, and his team received the Silver Award from the Hong Kong Electronic Industry Association for Outstanding Innovation and Technology Product (2000) for the Mobile and Online Vending EnableR (MOVER) solution. His biography has been published in Marquis' Who's Who in the World since 1998 and in Who's Who in Asia. 